\title{
Research on Comparison of Simulation and Experiment in Warpage Defects in Roll Forming Process of the Cap-Shaped Part Using a Five- Boundary Condition Forming Angle Distribution Function
}

\section{Mingyan Wang}

Shandong University of Science and Technology

\section{Zhengyang Feng}

Shandong University of Science and Technology

\section{Rui Wang}

Shandong University of Science and Technology

\section{Guosong Zhang}

Shandong University of Science and Technology

\section{Yuting Lv}

Shandong University of Science and Technology

\section{Shumei Lou}

Shandong University of Science and Technology

Chunjian Su ( $\square$ sdkjjd314@163.com )

Shandong University of Science and Technology

\section{Original Research}

Keywords: Roll Forming, Warpage, Five-boundary conditions, Forming angle

Posted Date: February 11th, 2021

DOl: https://doi.org/10.21203/rs.3.rs-175336/v1

License: (a) (i) This work is licensed under a Creative Commons Attribution 4.0 International License.

Read Full License 


\section{Research on Comparison of simulation and experiment in warpage defects in roll forming process of the cap-shaped part using a five-boundary condition forming angle distribution function}

Mingyan Wang ${ }^{1}$ Zhengyang Feng ${ }^{1}{ }^{2}$ Rui Wang ${ }^{1}$ Guosong Zhang ${ }^{1}$ Yuting Lv ${ }^{1}$

Shumei Lou ${ }^{1}$ Chunjian $\mathrm{Su}^{1 *}$

1) College of Mechanical and Electronic Engineering, Shandong University of Science and Technology, Qingdao, 266590, China

2) Co-first author

*Corresponding author

E-mail: sdkjdxjd314@163.com

Ethical Approval:

Not applicable

Consent to Participate:

Not applicable

Consent to Publish:

Not applicable

Authors Contributions:

Not applicable

Funding: 
The authors would like to acknowledge the financial support provided by the National Science Foudation of China (Grant No. 51705295), National Science Foudation of China (Grant No.51305241),Shandong Provincial Natural Science Foundation,China (ZR2018MEE022), and Support Program for Youth Innovation Technology in Colleges and Universities of Shandong Province (2019KJB015).

\title{
Competing Interests:
}

Not applicable

\section{Availability of data and materials:}

Not applicablef

\begin{abstract}
Warpage is one of the main defects in multi-pass roll forming. It manifests as the horizontal deviation in the sheet flange part after forming, impacting the final quality of the sheet. In this manuscript, we focus on the cap-shaped part of a small section profile. Considering the lack of previous scientific guidance on the distribution of forming angles - angles that lead to warpage defects and other defects reducing the quality of these products - a five-boundary condition distribution function of forming angle is proposed. We propose a mechanism for warpage defects in roll bending of a cap-shaped part based on this five-boundary condition forming angle distribution function. Furthermore, we examine the effects of forming angle, sheet thickness, and material yield strength on the warpage defects by examining the designed fluctuation of the edge wave, $\Delta z$, and the maximum deviation of the profile curves of the flange edge, $z^{\prime}$, to test this theory, roll bending experiments are compared to simulation.
\end{abstract}

Keywords:Roll Forming;Warpage;Five-boundary conditions;Forming angle

\section{Introduction}

Roll forming is a technique that feeds a metal sheet through sequential forming rolls such that the sheet is laterally bent in to a specific cross-sectional shape. This process is shown in Figure 1. 


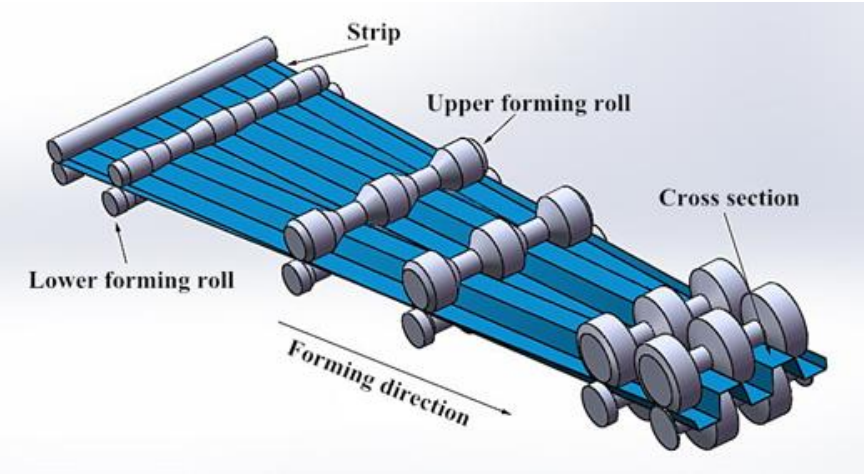

Fig. 1 Roll forming process

The bending angle is the most important processing parameter in the roll forming process. This bending angle is determined by production experience, and there is no strict theory to define the division of the bending angle. To optimize this process, the development of an effective bending angle division method is of value. To this end, Hirosh et al. [1] proposed that the edge deformation of the strip follows a cubic curve. This work shows that the horizontal plane projection trajectory at the end of the standing edge follows a cubic curve, proposing an ideal distribution of the bending angles. Bidabadi et al. have also studied this phenomenon, [2][3] evaluating the longitudinal bending of roll forming in a symmetrical U-shaped cross section both experimentally and numerically. These results show that the most important parameter is the forming angle. Furthermore, Han et al. [4] effectively utilized a B3-spline finite strip method to study the influence of molding parameters in cold rolling forming of channel section.

Warping is a common defect formed in multi-pass roll bending, as shown in Figure 2. Specifically, Tehrani et al. [5] believed that warpage is caused by changes in longitudinal strain on the sheet. To this end, Luo Xiaoliang et al. [6] have found, through numerical simulation, that during the bending process of high-strength steel rolls, certain material properties decrease the likelihood of warping, including: a larger yield strength, an increased strengthening coefficient, and a larger thickness anisotropy coefficient. Han et al. [4] found that the longitudinal strain in roll bending increases with plate thickness, larger deformation, and other parameters. By establishing a numerical simulation model, Wang Zhenxiao et al. [7] found that the deformation path in flexible roll bending has a large impact on the generation of warping. Moreover, with an increase in the number of rolls and the distance between passes, the side wave effect is weakened. Through 
numerical simulation, Li Yu et al. [8] evaluated the distribution of changes in stress and strain. At the same time, orthogonal testing shows that the flange height, sheet metal thickness, yield strength of sheet metal, and the number of forming paths all impact the formation and size of warpage. In conclusion, the roll forming process requires further study, specifically in regards to the control and reduction of warping through technological parameters.

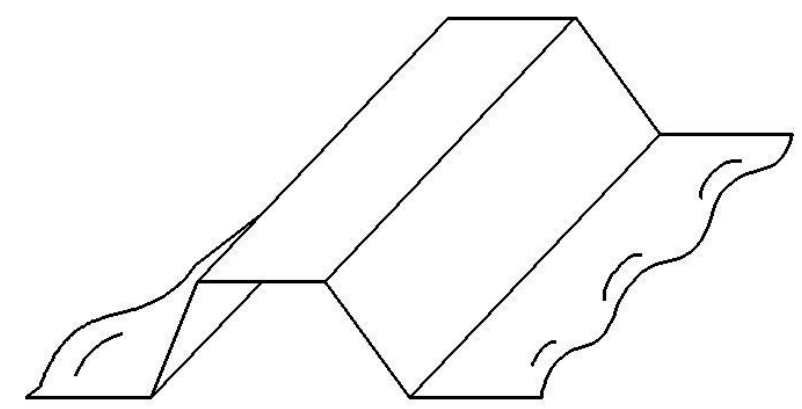

Fig. 2 Schematic diagram of edge wave as showing a hat-shaped defect

Before completing our study, we utilized production experience and theoretical analysis to propose a successful forming angle distribution function based on a five-boundary condition. To this end, we constructed a curve equation of the projection trajectory for the contour section edge in the horizontal plane. Simultaneously, we prove that when the forming angle interval of the first third of the forming process is $30 \% \times \theta_{0} \leq \theta_{N / 3} \leq 35 \% \times \theta_{0}$, the forming angle distribution is ideal $[9][10]$.

\section{Methodologies}

\subsection{Roll forming theory with five-boundary condition forming angle distribution function}

Bending angle (forming angle) is the most important and challenging issue in roll forming design. A well designed division of bending angle reduces stress concentration and resulting defects, such as longitudinal bending, edge tearing, and springback. By utilizing production experience and related theories, Professor Konai Hiroshi from Japan has proposed a bending angle distribution formula for roller forming [1]. This work suggests that when the projection track of the horizontal plane of the edge end follows a cubic curve, the bending angle distribution of plate is the ideal. This method of bending angle allocation is shown in Figure 3. 


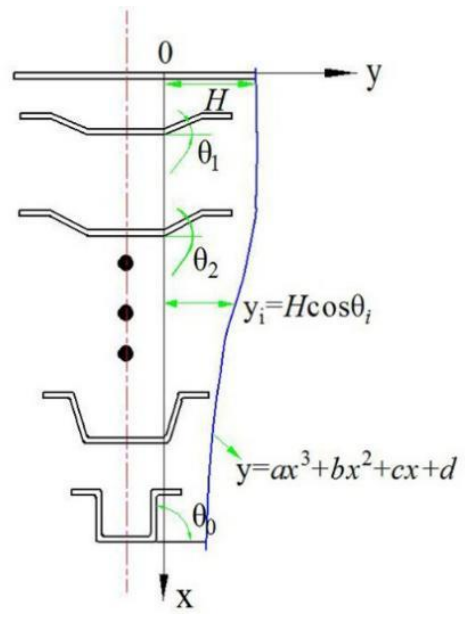

Fig. 3 The ideal projection track of the profile edge section in the horizontal plane follows a cubic curve.

As shown in Figure 3, assuming that the number of forming channels is $N$, the final bending angle of the vertical edge is $\theta_{0}$, the length of the vertical edge is $H$, and the bending angle of the $i$ th vertical edge is $\theta_{i}$, the expression of the cubic curve is as follows:

$$
y=A x^{3}+B x^{2}+C x+D
$$

This includes four-boundary condition equations:

$$
\left\{\begin{array}{c}
\frac{\mathrm{d} y}{\mathrm{~d} x}=0,(x=0) \\
\frac{\mathrm{d} y}{\mathrm{~d} x}=0,(x=N) \\
y=H,(x=0) \\
y=H \cos \theta_{0},(x=N)
\end{array}\right.
$$

By combining Equation (1) and boundary conditions (2), the following can be obtained:

$$
y=\frac{2 H\left(1-\cos \theta_{0}\right)}{N^{3}} x^{3}+\frac{3 H\left(\cos \theta_{0}-1\right)}{N^{3}} x^{2}+H
$$

When $x=i$ and $y=H \cos \theta_{i}$, the relationship between bending angle and forming pass, Equation (3) can be written as follows:

$$
\cos \theta_{i}=1+\left(1-\cos \theta_{0}\right)\left[2\left(\frac{i}{N}\right)^{3}-3\left(\frac{i}{N}\right)^{2}\right]
$$

By taking $I=1,2,3 \ldots N$ into Equation (4), the bending angle of each forming pass can be calculated. A bending angle distribution using a four-boundary condition model with nine passes is 
utilized in this study and shown in Table 1, where $i$ is the iterative forming pass, $\theta i$ is the bending angle, $\Delta \theta_{i}$ is the bending angle increment.

Table 1 The forming angle distribution of the four-boundary condition

\begin{tabular}{|c|c|c|c|c|c|c|c|c|c|}
\hline \multicolumn{10}{|c|}{ Forming passs } \\
\hline$i$ & 1 & 2 & 3 & 4 & 5 & 6 & 7 & 8 & 9 \\
\hline$\theta i$ & 15.0 & 29.1 & 42.2 & 54.3 & 65.4 & 75.0 & 82.7 & 88.0 & 90.0 \\
\hline$\Delta \theta i$ & 15.0 & 14.1 & 13.1 & 12.1 & 11.1 & 9.6 & 7.7 & 5.3 & 2.0 \\
\hline
\end{tabular}

\subsubsection{Simulation and optimization of forming angle distribution method based on five- boundary condition}

The bending angle allocation method based on four-boundary conditions has some regularity, resulting in a quantitative bending angle allocation, but it cannot guarantee that the distribution result is ideal as there are some limitations. Following practical production experience and statistical analysis, generally the bending angle for the first third of the process should not exceed $50 \%$ of the final bending angle, that is, $\theta_{N / 3} \leq 50 \% \times \theta_{0}$. To this end, a fifth boundary condition, $y_{N / 3}=H \cos \theta_{N / 3}$, can be applied to the bending angle distribution function with a four-boundary condition, resulting in the construction of a bending angle distribution function with a fiveboundary condition.

Assuming that the projection trajectory of the vertical edge of the horizontal plane is part of the quadric curve, the expression for constructing the quadric curve is as follows:

$$
y=A x^{4}+B x^{3}+C x^{2}+D x+E
$$

Thus, when $x=N / 3$, and $y=H \cos \theta_{N / 3}$, the five-boundary conditions of Equation (5) are: 


$$
\left\{\begin{array}{c}
\frac{\mathrm{d} y}{\mathrm{~d} x}=0,(x=0) \\
\frac{\mathrm{d} y}{\mathrm{~d} x}=0,(x=N) \\
y=H \cos \theta_{N / 3},(x=N / 3) \\
y=H,(x=0) \\
y=H \cos \theta_{0},(x=N)
\end{array}\right.
$$

At $x=i$ and $y_{i}=H \cos \theta_{i}$, by following the derivation process of the four-boundary condition in the previous section and utilizing Equations (5) and (6), the five-boundary condition bending angle distribution function of the $i$-th roller with bending angle $\theta_{i}$ can be obtained:

$$
\cos \theta_{i}=1+\frac{81 \cos \theta_{N / 3}-60}{4 N^{4}} i^{4}+\frac{-81 \cos \theta_{N / 3}+64}{2 N^{3}} i^{3}+\frac{81 \cos \theta_{N / 3}-72}{4 N^{2}} i^{2}
$$

According to former research [10], the optimal first third of the forming angle interval is $30 \% \times \theta_{0} \leq \theta_{N / 3} \leq 35 \% \times \theta_{0}$. Further optimization of the forming angle in the distribution interval yields $30 \% \times \theta_{0} \leq \theta_{N / 3} \leq 35 \% \times \theta_{0}$. When $\theta_{N / 3}=30 \% \times \theta_{0}, 31 \% \times \theta_{0}, 32 \% \times \theta_{0}$, $33 \% \times \theta_{0}, \quad 34 \% \times \theta_{0}, \quad 35 \% \times \theta_{0}$ and $i=1,2 \ldots N$, Equation (7) can be used to obtain the following results for each forming angle under different $\theta_{N / 3}$, as shown in Table 2:

Table 2 The forming angle distribution results for each pass when $\theta_{N / 3}$

\begin{tabular}{|c|c|c|c|c|c|c|c|c|c|c|c|}
\hline \multirow[t]{2}{*}{$\theta_{N / 3}$} & \multicolumn{11}{|c|}{ Forming pass } \\
\hline & $i$ & 0 & 1 & 2 & 3 & 4 & 5 & 6 & 7 & 8 & 9 \\
\hline \multirow{3}{*}{$35 \% \times \theta_{0}$} & $\theta_{i}$ & 0.00 & 8.96 & 19.70 & 31.50 & 43.85 & 56.28 & 68.21 & 78.82 & 86.77 & 90.00 \\
\hline & & & & & & & & & & & \\
\hline & $\Delta \theta_{i}$ & 0.00 & 8.96 & 10.74 & 11.80 & 12.35 & 12.43 & 11.93 & 10.61 & 7.95 & 3.23 \\
\hline \multirow{3}{*}{$34 \% \times \theta_{0}$} & $\theta_{i}$ & 0.00 & 8.35 & 18.85 & 30.6 & 43.02 & 55.59 & 67.71 & 78.53 & 86.68 & 90 \\
\hline & & & & & & & & & & & \\
\hline & $\Delta \theta_{i}$ & 0.00 & 8.35 & 10.5 & 11.75 & 12.42 & 12.57 & 12.13 & 10.82 & 8.14 & 3.32 \\
\hline \multirow{3}{*}{$33 \% \times \theta_{0}$} & $\theta_{i}$ & 0.00 & 7.71 & 17.98 & 29.7 & 42.19 & 54.91 & 67.22 & 78.25 & 86.59 & 90 \\
\hline & & & & & & & & & & & \\
\hline & $\Delta \theta_{i}$ & 0.00 & 7.71 & 10.27 & 11.72 & 12.49 & 12.71 & 12.32 & 11.03 & 8.33 & 3.41 \\
\hline $32 \% \times \theta_{0}$ & $\theta_{i}$ & 0.00 & 7.03 & 17.10 & 28.80 & 41.38 & 54.24 & 66.74 & 77.98 & 86.50 & 90.00 \\
\hline
\end{tabular}

( $\theta_{i}$ is the bending angle for the $i$ th pass, $\Delta \theta_{i}$ is the increment of the bending angle) 


\begin{tabular}{|c|c|c|c|c|c|c|c|c|c|c|c|}
\hline & $\Delta \theta_{i}$ & 0.00 & 7.03 & 10.07 & 11.70 & 12.58 & 12.86 & 12.50 & 11.24 & 8.52 & 3.50 \\
\hline \multirow{3}{*}{$31 \% \times \theta_{0}$} & $\theta_{i}$ & 0.00 & 6.30 & 16.19 & 27.90 & 40.57 & 53.59 & 66.28 & 77.72 & 86.41 & 90.00 \\
\hline & & & & & & & & & & & \\
\hline & $\Delta \theta_{i}$ & 0.00 & 6.30 & 9.89 & 11.71 & 12.67 & 13.01 & 12.69 & 11.44 & 8.70 & 3.59 \\
\hline \multirow{2}{*}{$30 \% \times \theta_{0}$} & $\theta_{i}$ & 0.00 & 5.50 & 15.27 & 27.00 & 39.78 & 52.95 & 65.82 & 77.46 & 86.33 & 90.00 \\
\hline & $\Delta \theta_{i}$ & 0.00 & 5.51 & 9.76 & 11.73 & 12.78 & 13.17 & 12.88 & 11.64 & 8.87 & 3.67 \\
\hline
\end{tabular}

Copra was used to simulate the roll bending forming process. First, according to the results in Table 2, developed using the five-boundary condition of roller bend forming process simulation, the main performance parameters of the material as shown in Table 3. Key shaping parameters entered into the software DataM Copra RF v2005 SR1 include: (1) the calculation party was set to DIN6935 (2) the simulation method was the Hauschild method (3) the number of racks was 10 (4) rack spacing was $500 \mathrm{~mm}$, etc.

Table 3 Mechanical properties of Q235 steel

\begin{tabular}{|c|c|c|c|c|c|}
\hline \multirow[t]{2}{*}{ material } & $\begin{array}{l}\text { Elastic } \\
\text { Modulus }\end{array}$ & Mass density & $\begin{array}{c}\text { Yield } \\
\text { Strength }\end{array}$ & $\begin{array}{c}\text { Poisson's } \\
\text { ratio }\end{array}$ & $\begin{array}{l}\text { Sheet } \\
\text { thickness }\end{array}$ \\
\hline & $E(\mathrm{GPa})$ & $\rho\left(\mathrm{kg} / \mathrm{m}^{3}\right)$ & $\delta \mathrm{s}(\mathrm{MPa})$ & $\mu$ & $t(\mathrm{~mm})$ \\
\hline Q235 & 212 & $7.86 \mathrm{E}+03$ & 235 & 0.288 & 1.5 \\
\hline
\end{tabular}

The resulting simulation of the peak longitudinal strain is shown in Figure 4.
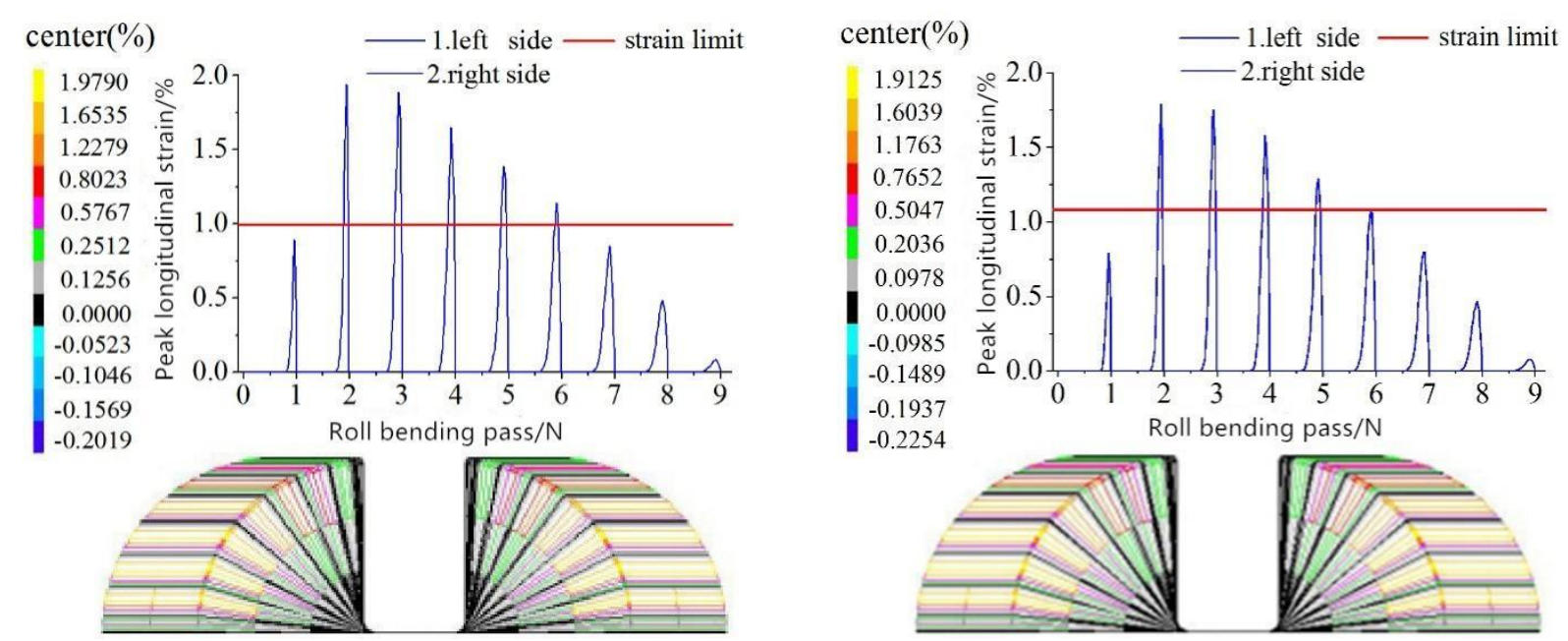
(a) $30 \% \times \theta_{0}$

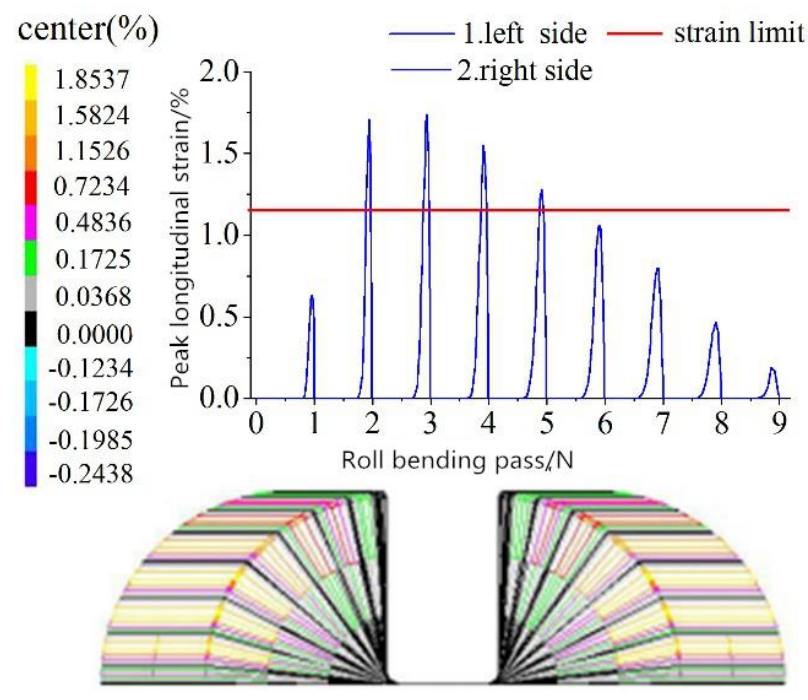

(c) $32 \% \times \theta_{0}$

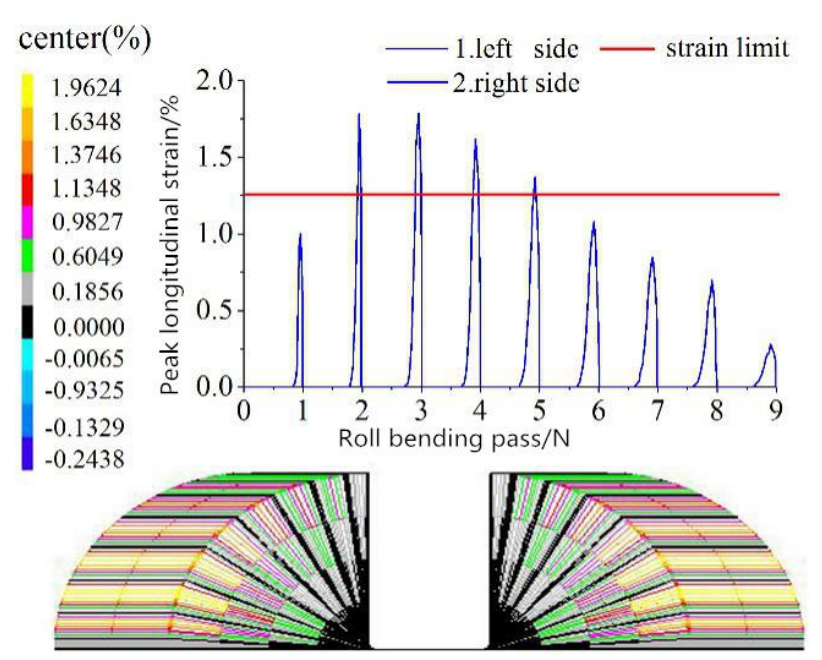

(e) $34 \% \times \theta_{0}$ (b) $31 \% \times \theta_{0}$

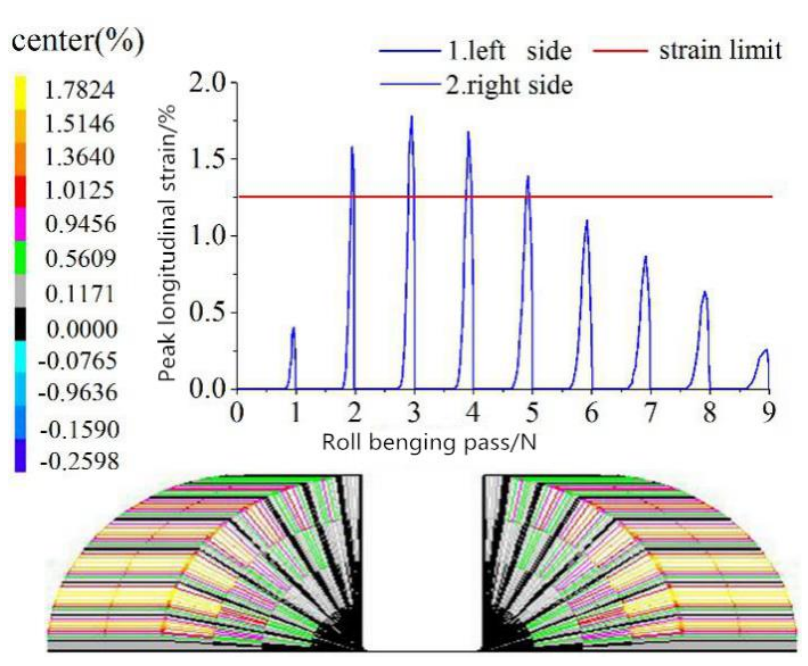

(d) $33 \% \times \theta_{0}$

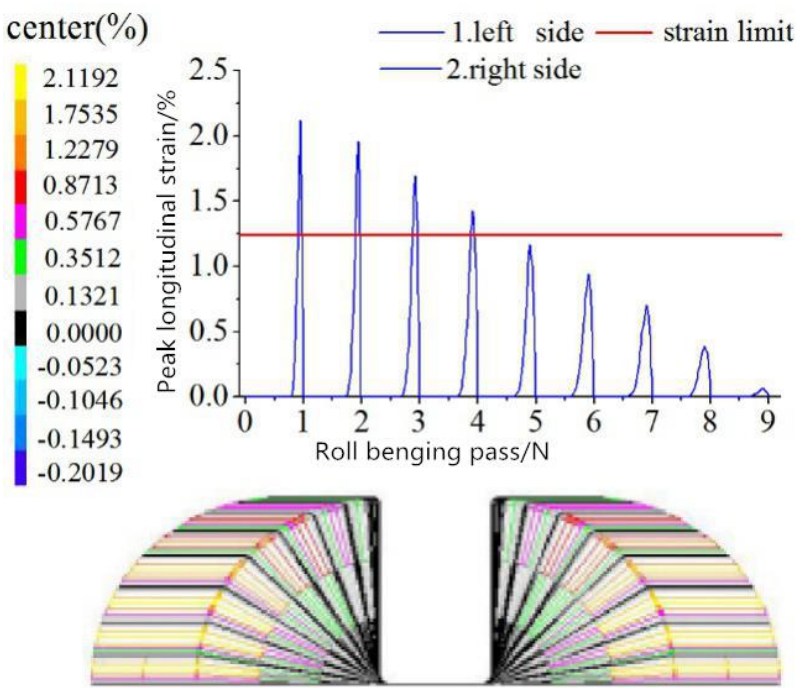

(f) $35 \% \times \theta_{0}$

Fig. 4 Simulation of peak longitudinal strain using five-boundary condition method

Thus, through this simulation, the longitudinal strain of roll bending based on the fiveboundary conditions, as shown in Figure 4, was developed, showing the peak longitudinal strain between tracks.

Similarly, by utilizing the above parameters and Table 1, the peak longitudinal strain of roll forming based on four-boundary condition shown is shown in Fig 5. 


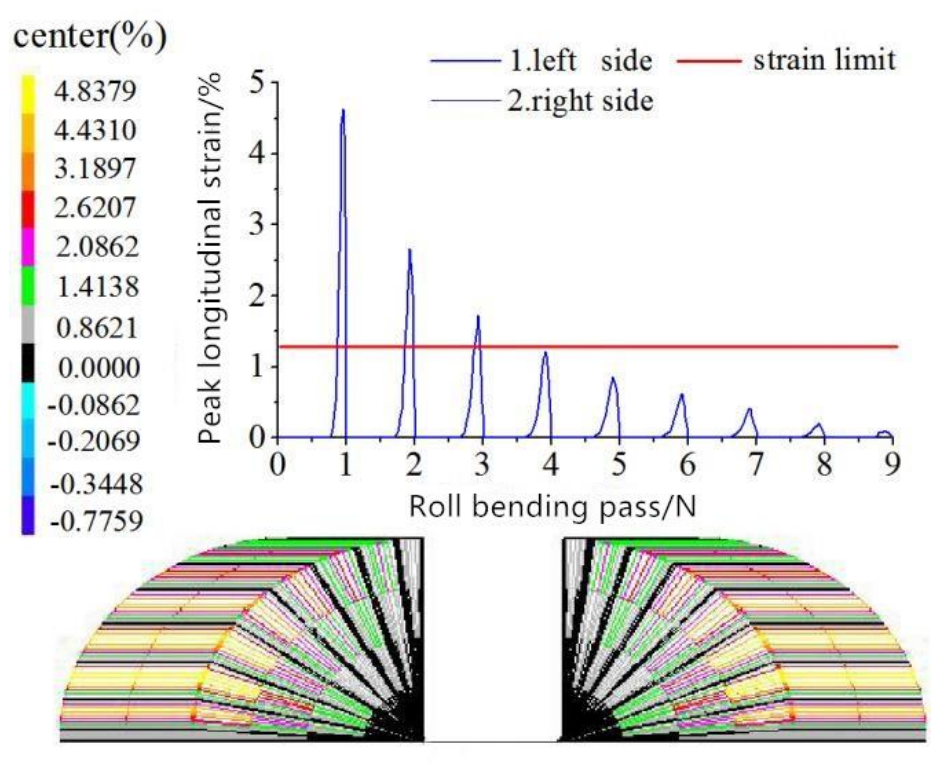

Fig. 2.3 Simulation of peak longitudinal strain based on four-boundary condition

In order to analyze the simulation results of peak longitudinal strain, Figure .6 compares the peak longitudinal strain with the different boundary conditions. When $\theta_{N / 3}=50 \% \times \theta_{0}$, the trends of the four-boundary condition and the five-boundary condition is consistent. However, for the four-boundary condition method, the maximum peak longitudinal strain occurs at the first pass, that is, at the bite stage of forming is too high, the deformation too large, and the forming angle too big, features that are not conducive to the bite of sheet metal. Among the four fold lines, the $33 \%$ $\times \theta_{0}$ fold line is gentler than the other three fold lines, resulting in a distribution that is more reasonable. 


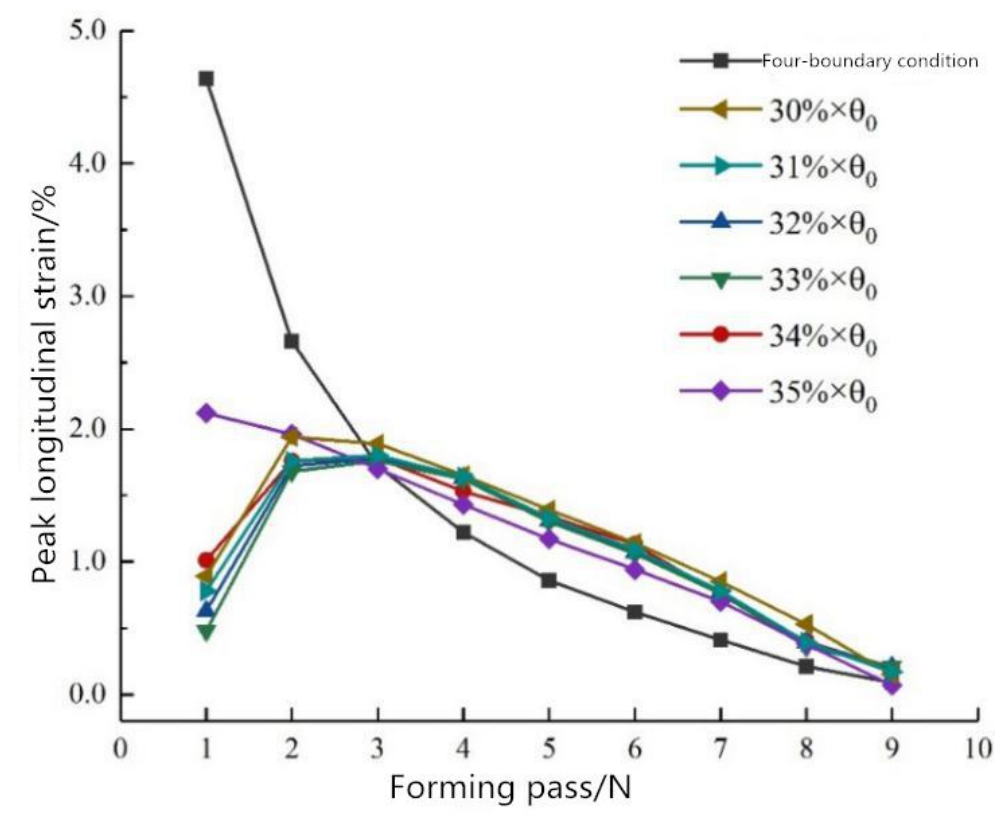

Fig. 6 Comparison of peak longitudinal strain between passes based on different boundary conditions

The above analysis shows that the method with four-boundary condition cannot guarantee the best forming angle distribution result, thus the five-boundary condition method obtains the optimal forming angle allocation result.

Based on these results, we selected $\theta_{N / 3}=33 \% \times \theta_{0}$ for experimental analysis. By applying $I$ $=1,2 \ldots, N$ into Equation (7), we obtained the distribution of the forming angles for each pass and the resulting distribution is shown in Table 4.

Table 4 Forming angle distribution results for $\theta_{N / 3}=33 \% \times \theta_{0}$

\begin{tabular}{|c|c|c|c|c|c|c|c|c|c|c|}
\hline pass & 0 & 1 & 2 & 3 & 4 & 5 & 6 & 7 & 8 & 9 \\
\hline The forming Angle $\left(\theta_{i}\right)$ & 0.00 & 7.71 & 17.98 & 29.7 & 42.19 & 54.91 & 67.22 & 78.25 & 86.59 & 90 \\
\hline $\begin{array}{l}\text { Increment of forming Angle } \\
\qquad\left(\Delta \theta_{i}\right)\end{array}$ & 0.00 & 7.71 & 10.27 & 11.72 & 12.49 & 12.71 & 12.32 & 11.03 & 8.33 & 3.41 \\
\hline
\end{tabular}

\subsection{Abaqus Finite element simulation}

This study uses the software Abaqus to simulate the sheet roll forming process. The mechanical properties of the two materials studied are shown in Table 5, yielding the stress-strain curve shown in Figure 7. Figure 8 shows an established finite element model of roll forming, of 
which the grid of the sheet is shown in Figure 9. To improve accuracy of this simulation, the mesh is further refined in the bending area.

Table 5 Mechanical properties of materials

\begin{tabular}{cccccc}
\hline & Elastic & Mass & Yield & Poisson's & Tensile \\
material & Modulus & density & Strength & ratio & Strength \\
& E $(\mathrm{GPa})$ & $\rho\left(\mathrm{kg} / \mathrm{m}^{3}\right)$ & $\sigma_{\mathrm{s}}(\mathrm{MPa})$ & $\mu$ & $\sigma_{\mathrm{b}}(\mathrm{MPa})$ \\
\hline Q235 & 212 & $7.86 \mathrm{E}+03$ & 235 & 0.288 & 450 \\
DP780 & 218 & $8.0 \mathrm{E}+03$ & 578 & 0.3 & 886 \\
\hline
\end{tabular}

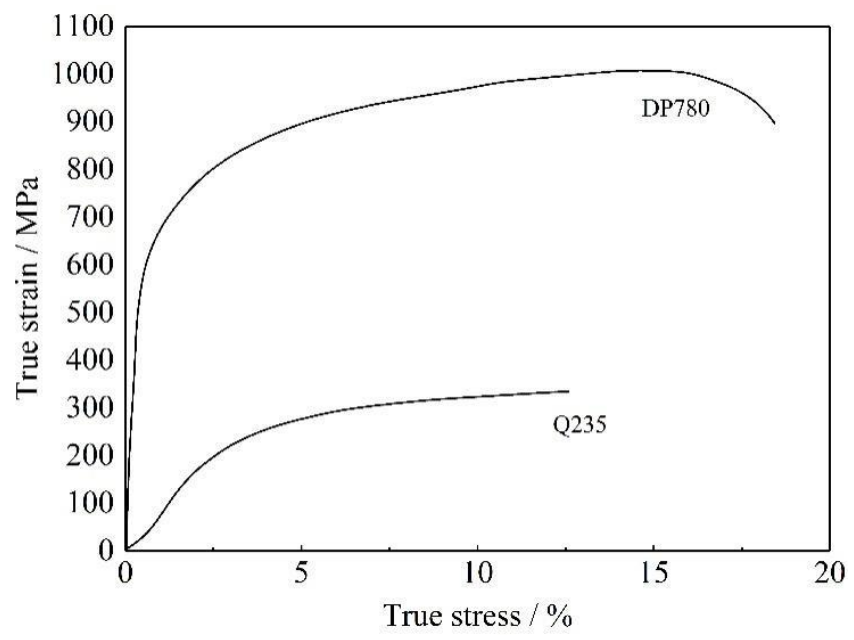

Fig. 7 True stress-strain curve

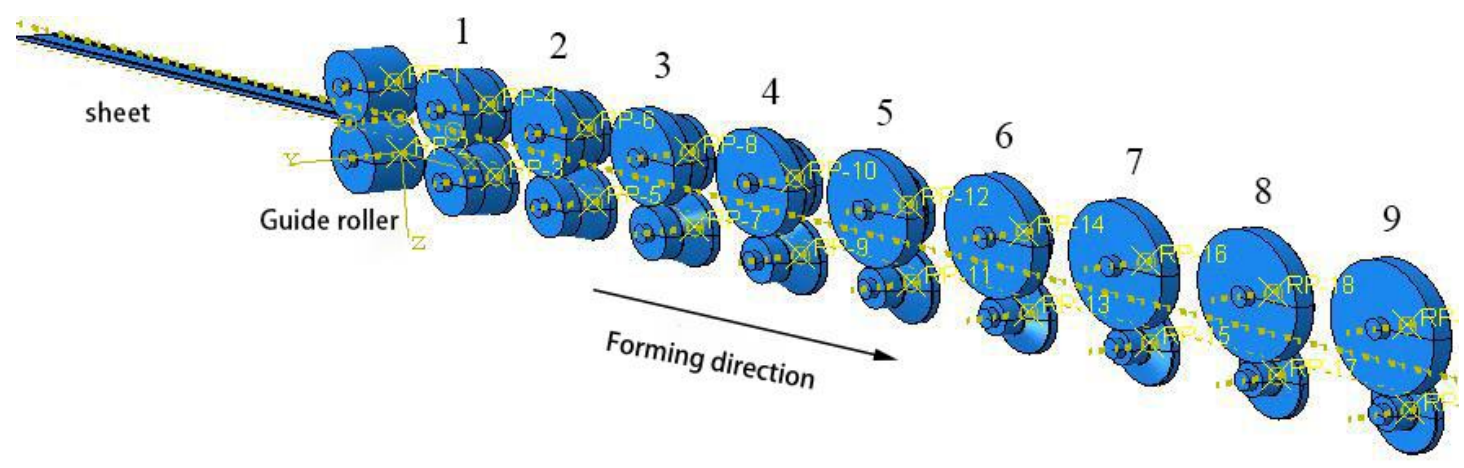

Fig. 8 The finite element model of roll forming 


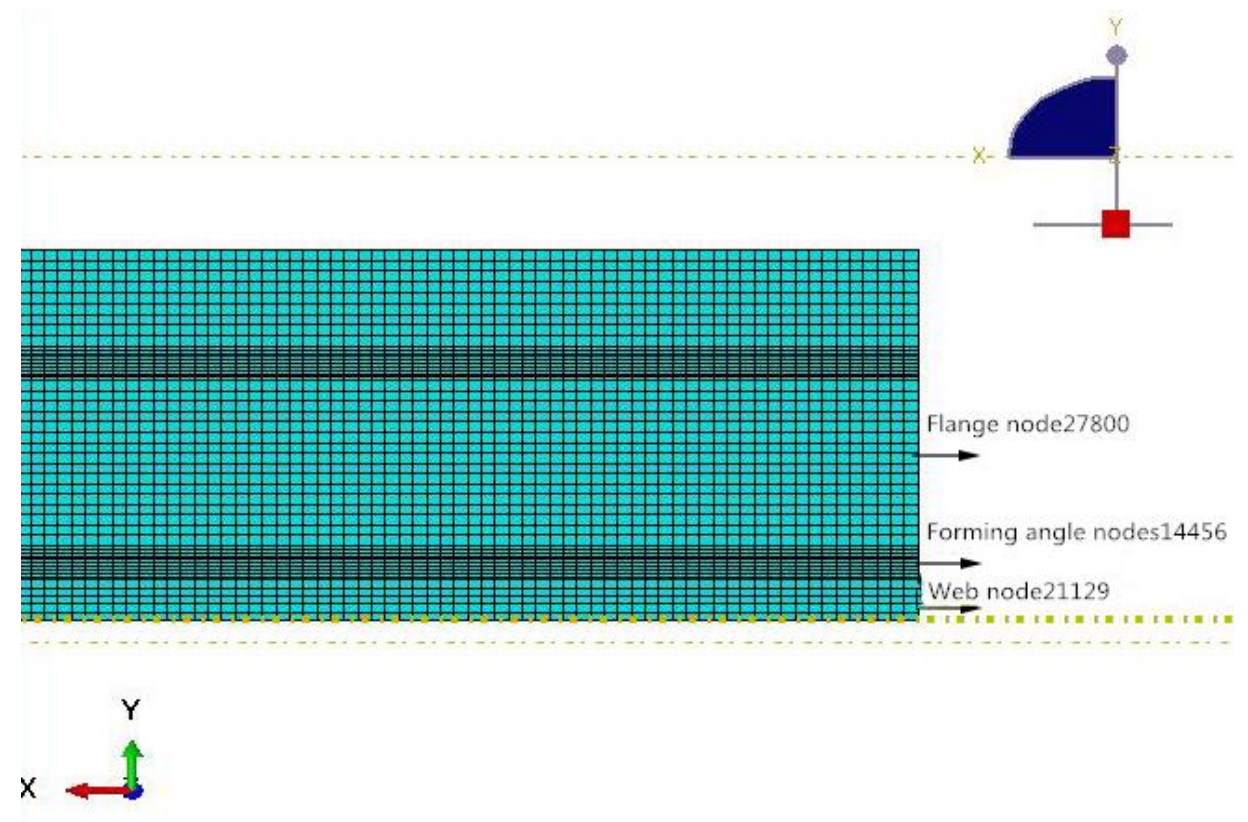

Fig.9 the grid of the sheet

\subsection{Experiment}

\subsubsection{Roll forming process experiment}

Figure 10 shows the roll forming set-up used in this study. Because the cross section of the hat-shaped piece is relatively simple, the machine is set to match the upper and lower rolls with 9 passes.

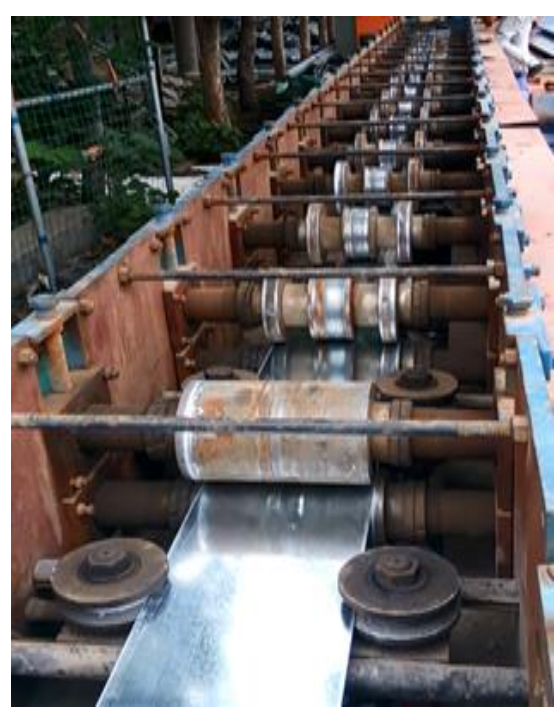

(a) Feed direction

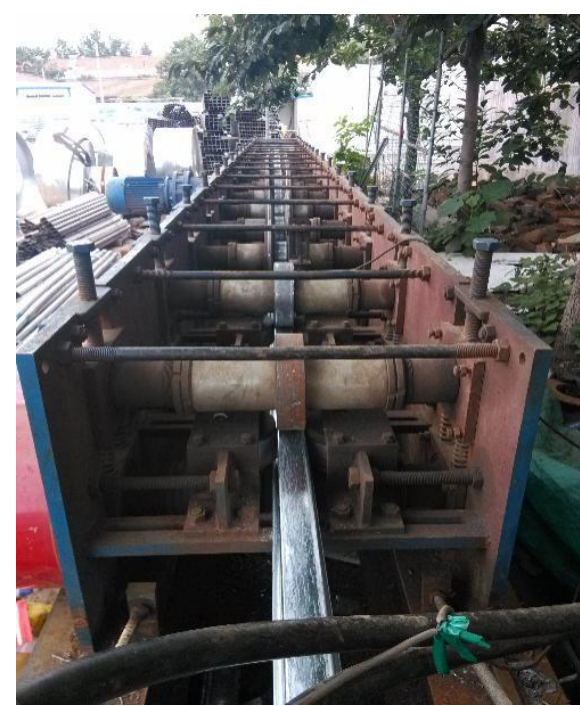

(b) Forming direction

Fig. 10The experimental equipment 


\subsubsection{Warpage measurement method of the cap-shaped part}

The hat-shaped piece obtained by the experiment is shown in Figure 11, and the forming quality is different under different forming parameters. In order to experimentally verify the impact of different forming parameters on the numerical simulation in the previous section, it is necessary to evaluate the warpage fluctuation at the flange edge through the maximum deviation of the $z$-axis coordinates. This value is measured as discussed below.

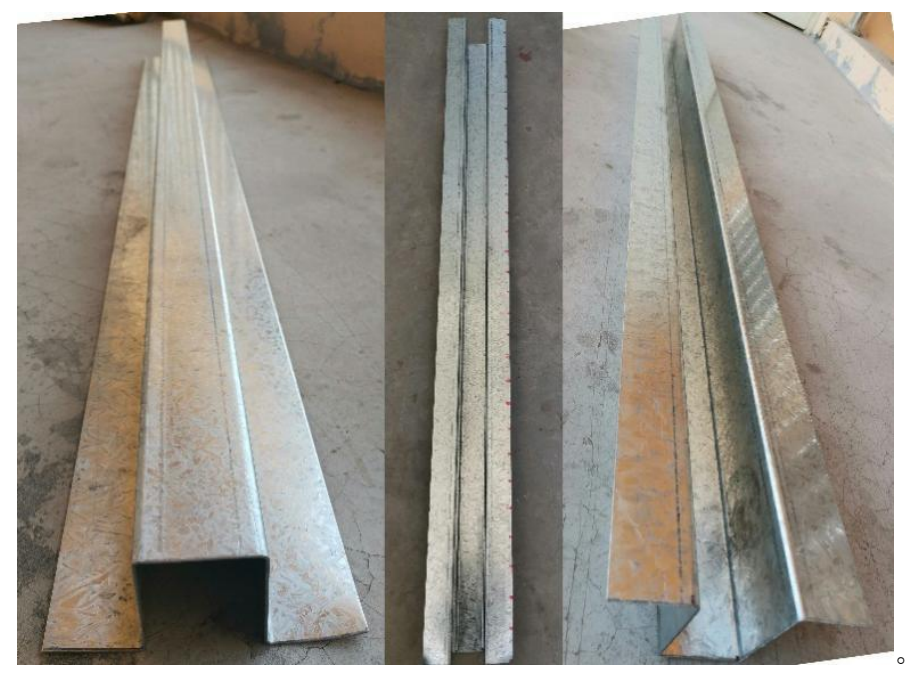

Fig. 11 Roll-formed hat-shaped

As shown in Figure 12, the longitudinal coordinate of the test point is marked with a red marker on the edge of the unformed sheet. The number of points is consistent with the simulated collection points. In the finite element simulation, the middle part of the web was placed at the beginning of the plate and is set as the coordinate origin, thus the experimentally formed hat-shaped web is placed on the horizontal test bench, and a caliper is used to measure the height from each measuring point to the test bench. This measurement determines the $z$-coordinate of the point, corresponding to the $z$-direction displacement in the simulation. Each measurement point is collected twice, and the average value is reported. 


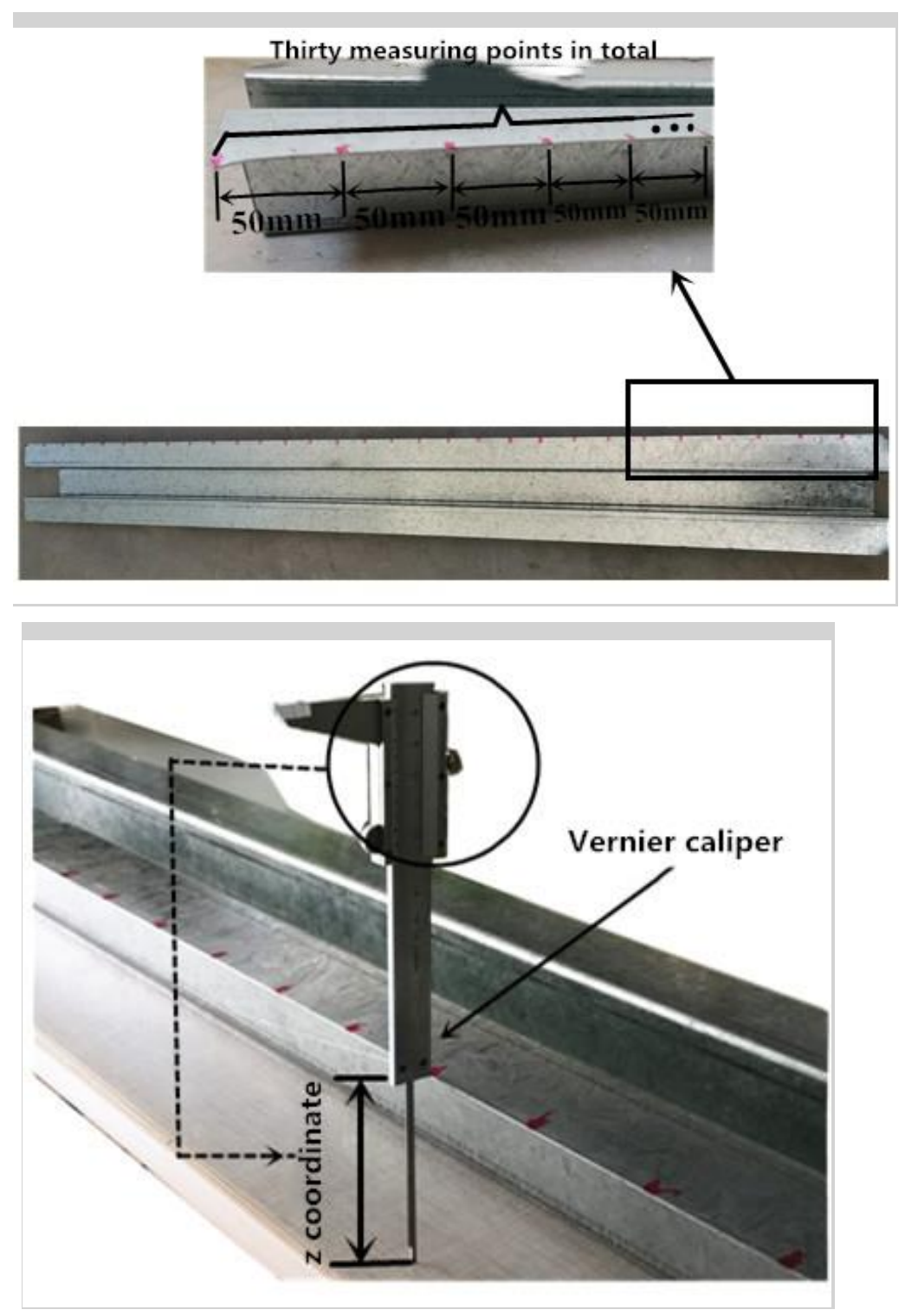

Fig. 12 Measuring point of roll-formed hat-shaped and measurement method

The z-direction coordinates obtained by experiment are compared with the simulated values obtained in the previous section.

\subsection{Roll forming warpage evaluation standard}

In the hat-shaped piece, the warpage reflects the differences between the vertical coordinates of the edge of the flange. Based on this, Figure 13 was developed to schematically show the warpage evaluation standard. To extract the node coordinates $\left(x_{i}, z_{i}\right)$ of the edge, the average of $z_{i}$ was taken to determine $\bar{z}$, by determining the standard deviation of the difference between $z_{i}$ and $\bar{z}$, the $\mathrm{z}$-coordinate of each node was calculated relative to the average $\bar{z}$. The maximum deviation 
is used as the evaluation index of warpage, and the calculation of the standard deviation is shown in Equation (11).

$$
\left\{\begin{array}{c}
\Delta z=\sqrt{\frac{1}{N} \sum_{i=1}^{N}\left(z_{i}-\bar{z}\right)^{2}} \\
z^{\prime}=\left|z_{i}-\bar{z}\right|_{\max }
\end{array}\right.
$$

In the Equation, $\Delta z$ is the standard deviation of the difference between $z_{i}$ and $\bar{z}$ of each node, $z^{\prime}$ is the maximum deviation between the $z$-coordinate of each node and the average $\bar{z}, N$ is the number of flange edge nodes, $y_{i}(I=1,2,3 \ldots N)$ is the $z$-coordinate of each node, and $\bar{z}$ is the average value of the z-coordinate of each node. In summary, the larger the value of $\Delta z$ and $z^{\prime}$, the greater the coordinate difference, indicating increased warpage.

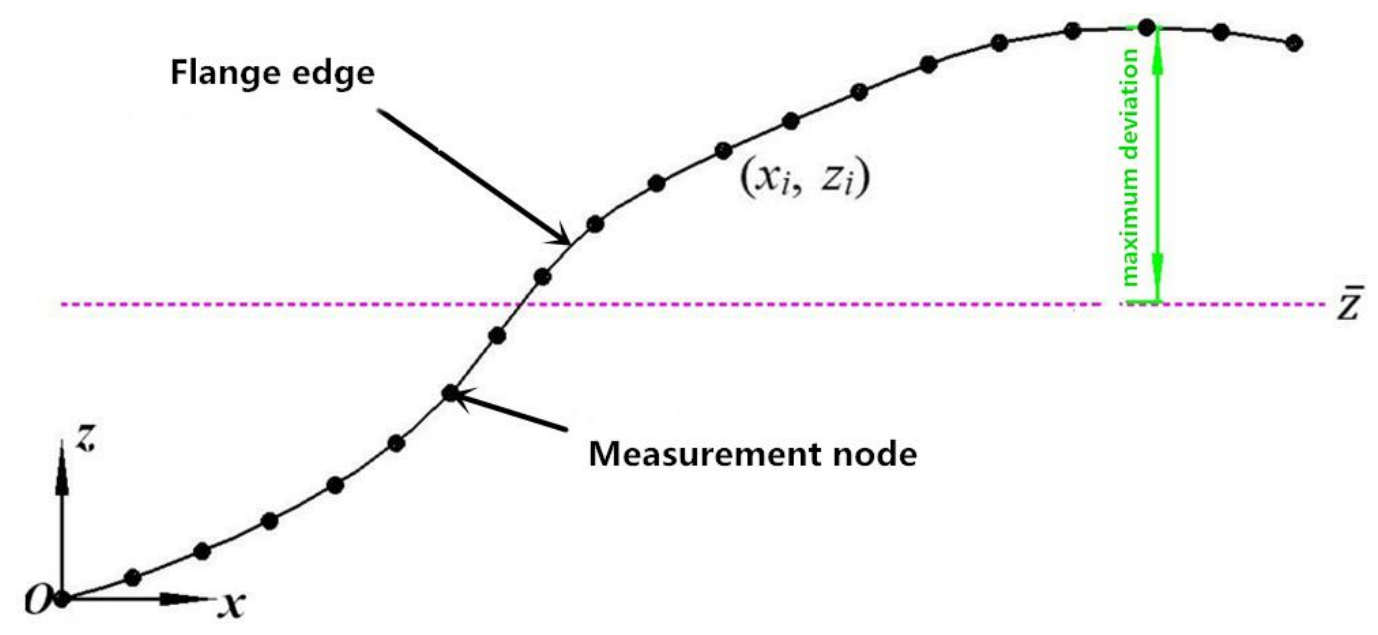

Fig.13 Schematic for evaluating edge wave

\section{Results and discussion}

3.1 Simulation analysis on the formation mechanism of warpage defects (Analysis of the formation mechanisms of edge wave)

Li Yu et al. [8] analyzed the stress and strain of thin-walled channel steel parts after roll forming to make the flange edge show plastic instability, producing a side wave. In contrast, our analysis of the stress and strain generated in the roll forming process of hat-shaped sheet metal parts explores the mechanisms of warpage in hat-shaped sheet metal parts. 
Based on the numerical simulation cloud diagram in Section 2, it can be seen that there are specific warping defects at the area of the forming angle. The key difference from Section 2 is that warping mainly occurs at the flange edge, thus research should be shifted from the area of forming angle in Section 2 to the flange edge.

In order to match observation with analysis in accordance with the forming law of the symmetrical section profile, the $1.5 \mathrm{~mm}$ thick Q235 steel with the same parameters as in the previous section with the five-boundary condition is again subjected to numerical simulation of roll forming, and the new cloud diagram showing the stress distribution of hat-shaped piece after forming is shown in Figure 14.

It can be seen from Figure 14(a) that the Mises equivalent stress distribution is not uniform in the entire hat-shaped piece, but in general, the closer a point is to the forming corner, the greater the equivalent stress. At the front end of the hat-shaped piece, the edge of the flange has a relatively obvious downward slope, which is related to the unstable nature of the front end of the sheet. In the middle and rear regions of the hat-shaped piece, more obvious warping can be observed, and at the place where the equivalent stress is small (blue), the flange is inclined upward; whereas, where the equivalent residual stress is larger (green), the flange slopes downward. As mentioned earlier, the Mises equivalent stress is determined by the longitudinal stress, S11, and the transverse stress, S22, at the flange. However, as can be seen from Figure 14(c), the transverse stress, S22, at the flange of the part is distributed more uniformly, while in Figure 14(b), the longitudinal stress, S11 (that is, the x-direction stress) at the flange is unevenly distributed. For Figure 3.1(b), the smaller regions (green) show the flange legs are inclined upward; in contrast, the larger part of S11 (red), the flange is inclined downward, which is consistent with the law found on the Mises chart. Thus, it can be concluded that the warpage is caused by the uneven distribution of the longitudinal residual stress, S11, at each point of the flange edge along the longitudinal direction (forming direction) of the part. The more uneven the distribution of the longitudinal residual stress, S11, becomes, the greater the longitudinal residual tension and compression stress gap are, thus resulting in more serious degrees of warpage. 


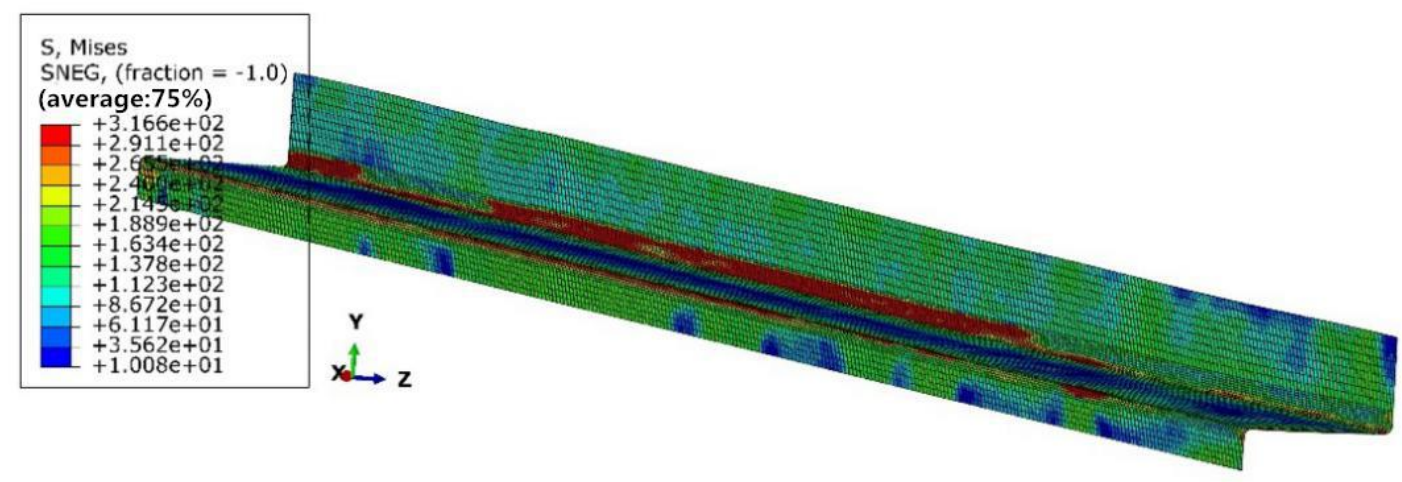

(a) Mises equivalent stress

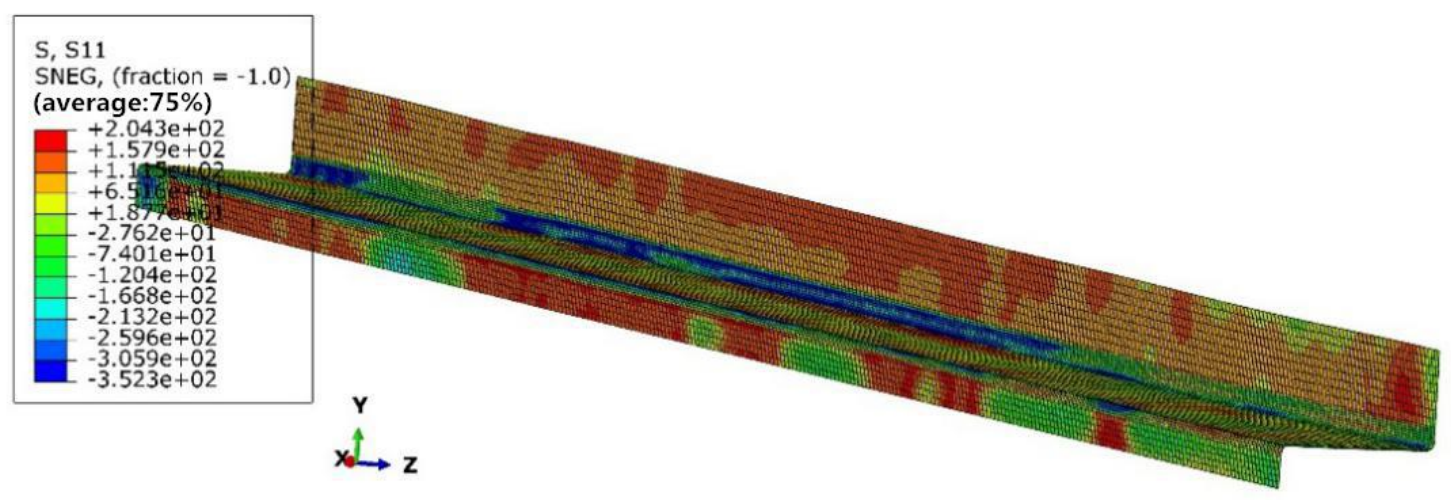

(b) Longitudinal stress, S11

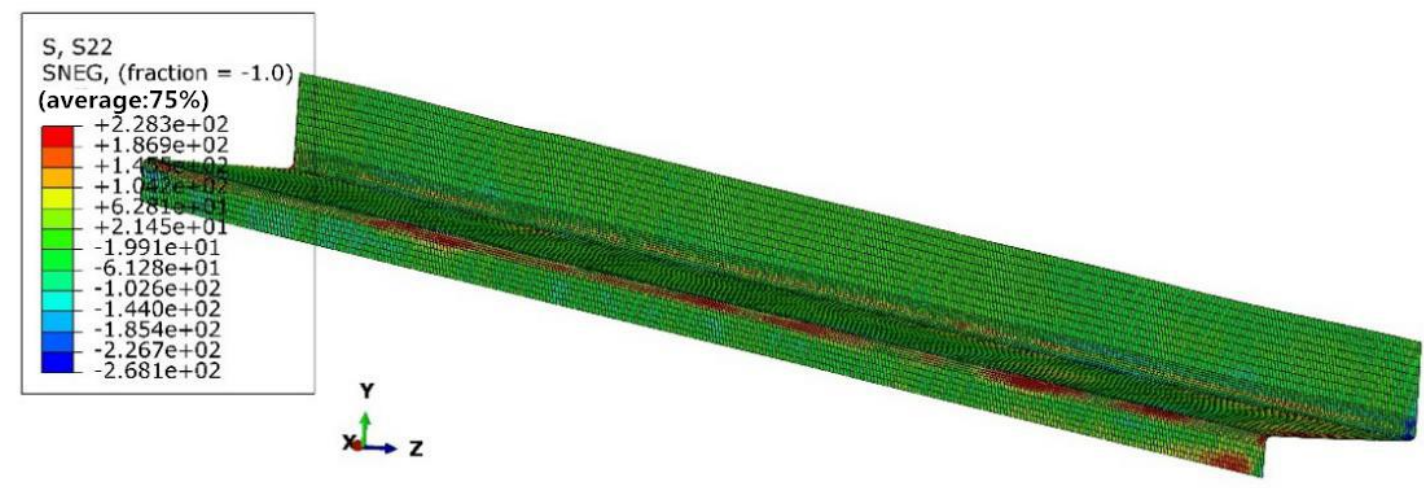

(c) Transverse stress, S22

Fig.14 Stress contour of hat-shaped piece 


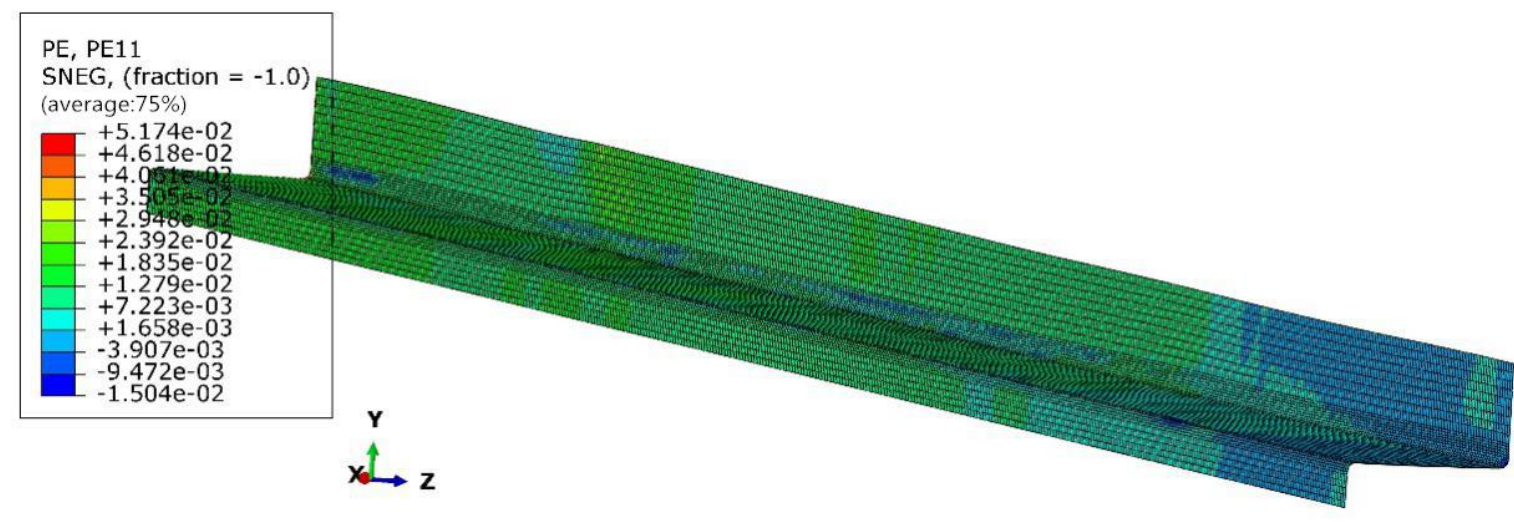

(a) Longitudinal strain, PE11

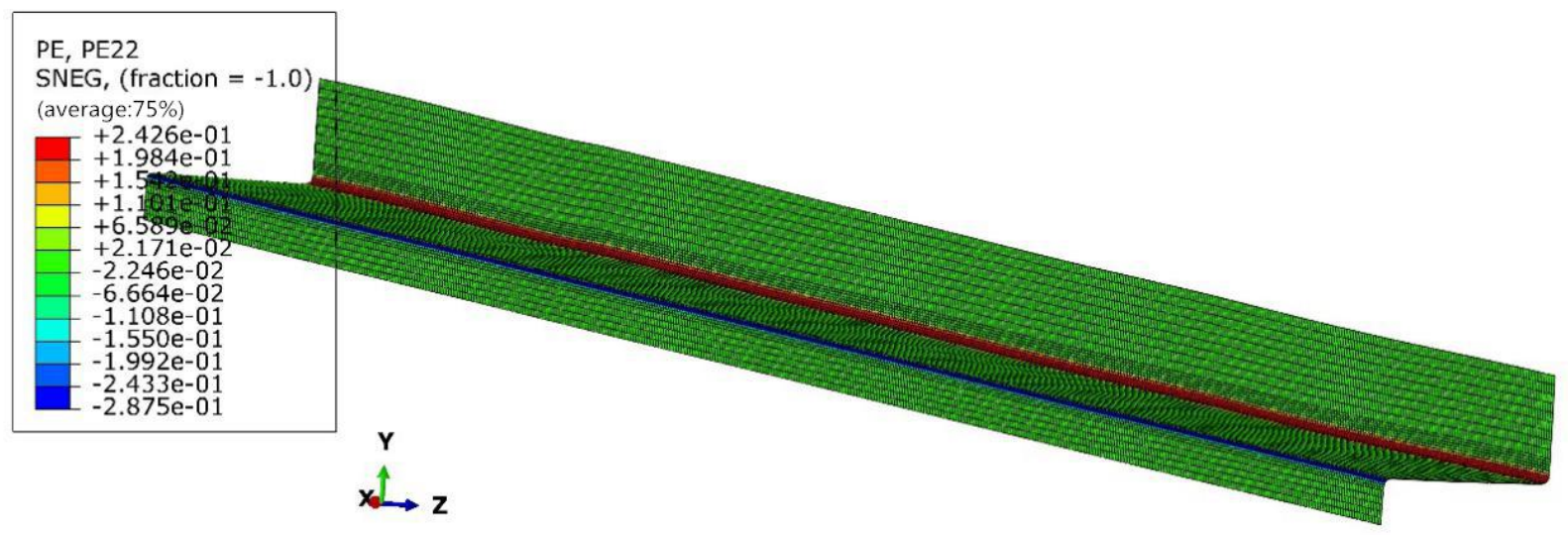

(b) Lateral strain, PE22

Fig.15 Plastic strain contour of hat-shaped piece

As seen above, the severity and distribution of warpage in the hat-shaped piece are related to the residual stress distribution at the flange part, and the residual stress is related to the degree of plastic deformation caused during the roll forming process. The resulting plastic strain contour of the hat-shaped piece with the same forming parameters is shown in Figure 15. By extracting the longitudinal strain, PE11, at the flange edge in Figure 3.1(a) we developed Figure 16. Figure 16 shows both the contour curve of the hat-shaped piece and the curve of the longitudinal strain, PE11, with respect to the longitudinal $\mathrm{x}$ coordinate. 


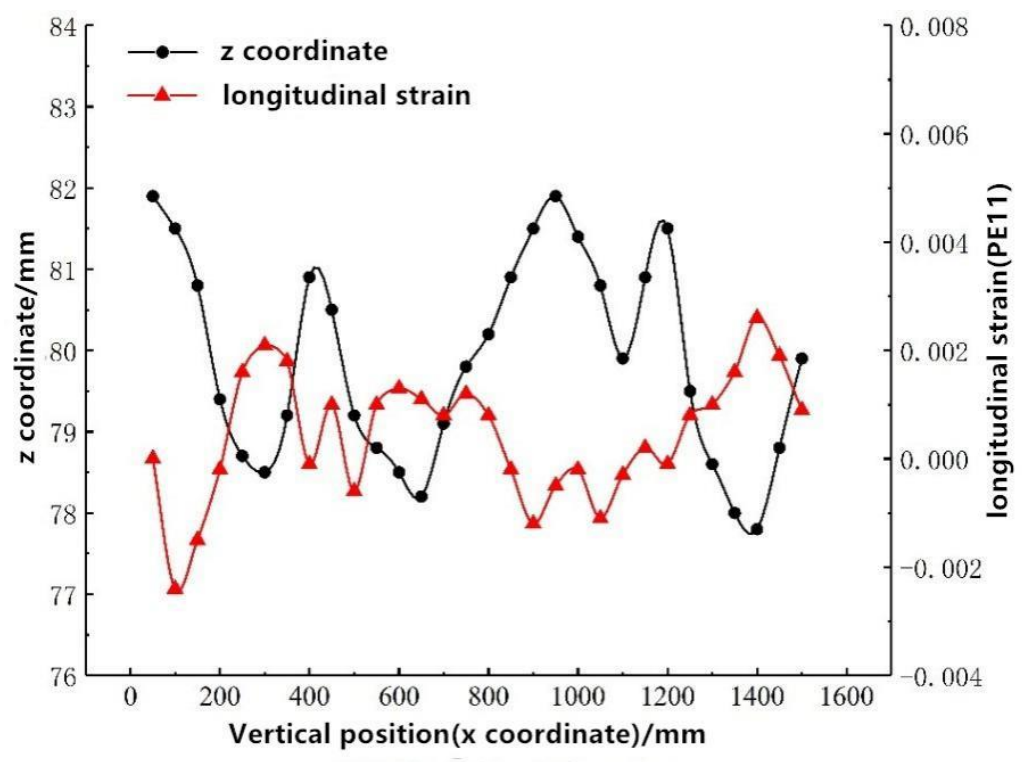

Fig.15 Curve of longitudinal strain, PE11, at flange edge as compared to the flange profile curve

As can be seen from Figure 16, the longitudinal strain, PE11, is unevenly distributed. The fluctuation law of the flange edge can be directly observed: at the exit of the hat, the $z$-coordinate of the flange edge is relatively large; in the middle and rear sections, the $z$-coordinate floats between $78 \mathrm{~mm}-82 \mathrm{~mm}$, that is, warping occurs. By comparing the two curves in Figure 16, it is seen that, in general, the sheet is plastically stretched in the longitudinal direction when the longitudinal strain, PE11, is positive. By evaluating the flange profile curve, we observe that as the $z$-coordinate is smaller, the flange edge bends downward. When the longitudinal strain, PE11, is small, the edge of the flange is inclined upward. In conclusion, the shift in longitudinal strain is consistent with the flange edge profile of the hat-shaped piece.

By completing this stress and strain analysis, we have determined that, under the joint action of longitudinal stress and longitudinal plastic strain, the plastic instability of the flange edge results in warping defects.

\subsection{Analysis of simulated and experimental results considering the influence of distribution methods on warpage defects}

The simulated and experimental parameters in this section are shown in Table 6. This section focuses on evaluating the z-coordinate and the shift of $\Delta z$ after the sheet is fully formed. 


\begin{tabular}{lll}
\hline material & Sheet thickness $(\mathrm{mm})$ & Angle distribution method \\
\hline $\mathrm{Q} 235$ & 1.5 & Five-boundary condition \\
& & Four-boundary condition \\
& Forming angle increment $10^{\circ}$ \\
\hline
\end{tabular}

Figure 17 shows the contour of the $z$-direction displacement as calculated utilizing different forming angle distribution methods. From this, it can be seen that the forming quality of the flange edge of the hat-shaped piece under the five-boundary condition is superior to other distribution methods.

In order to intuitively observe fluctuations of the flange edge of the hat-shaped piece, the postprocessing module collects the $(x, z)$ coordinates of each node in simulation. After completing the experiment, the module collects the coordinate data of each node $(x, z)$ of the flange edge,organizing records. Then, according to the data, the module draws the contour curve of the longitudinal nodes of the flange edge of the hat, as shown in Figure 18.

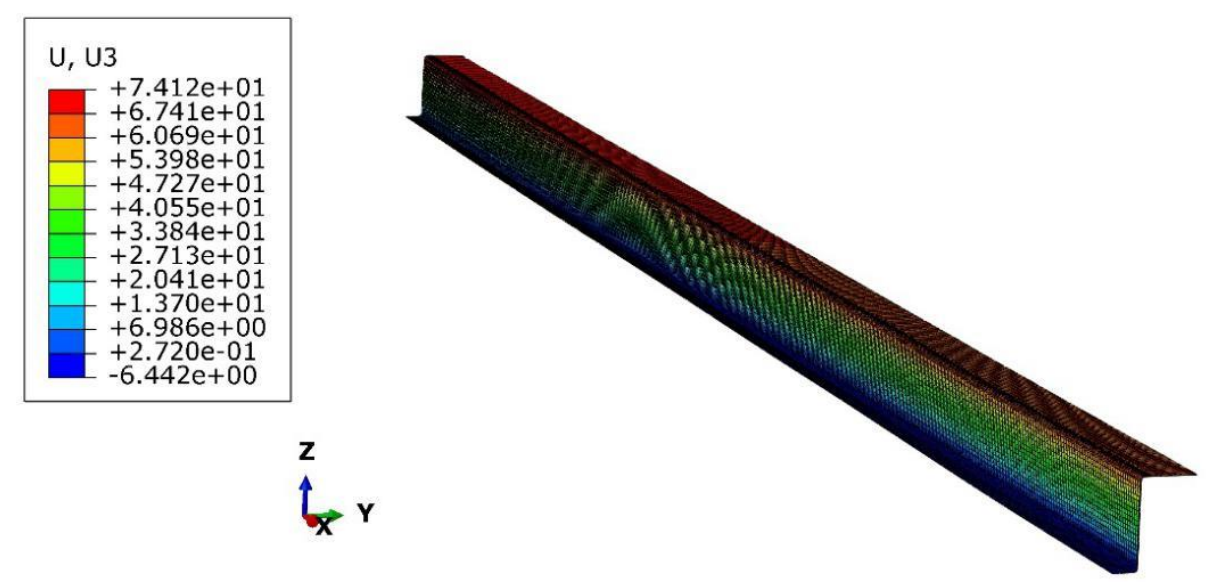

(a) Contour of the z-direction displacement using the five-boundary conditions function 


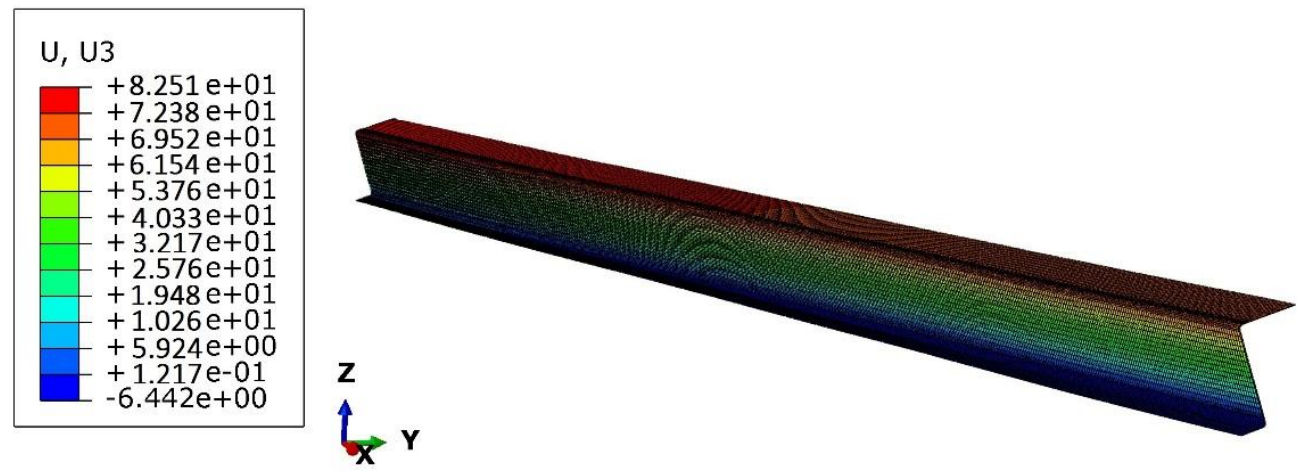

(b) Contour of the z-direction displacement using the four-boundary conditions function
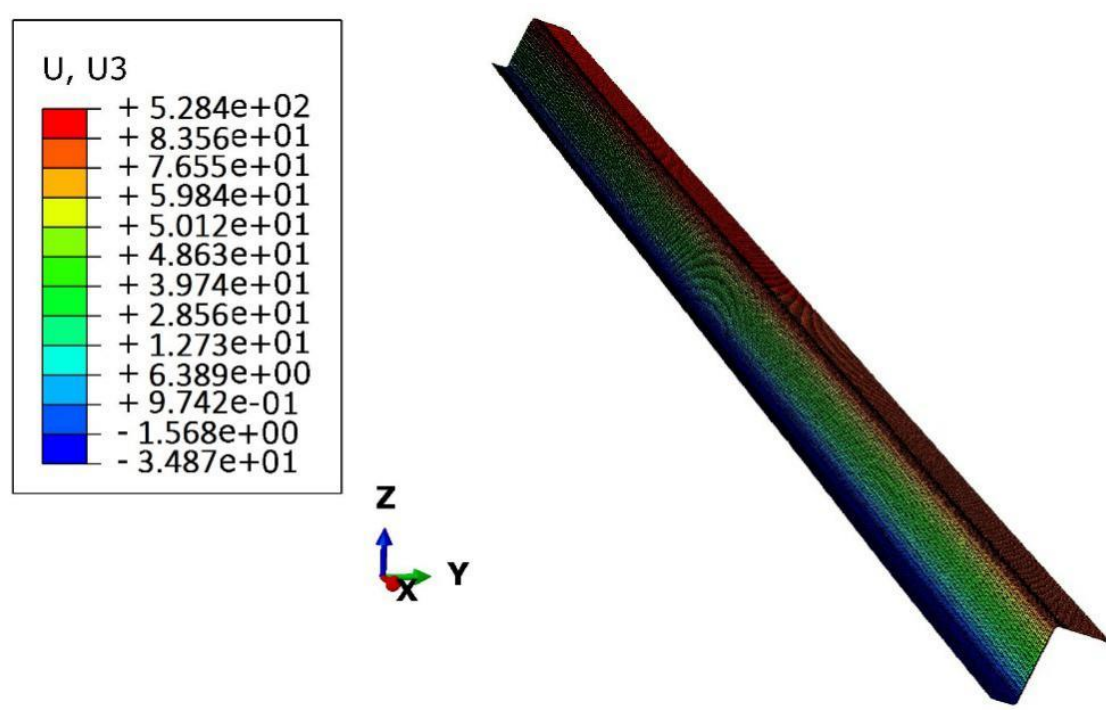

(c) Contour of the z-direction displacement using uniform forming angle distribution

Fig.17 Contour of z-direction displacement under different forming angle distribution methods 


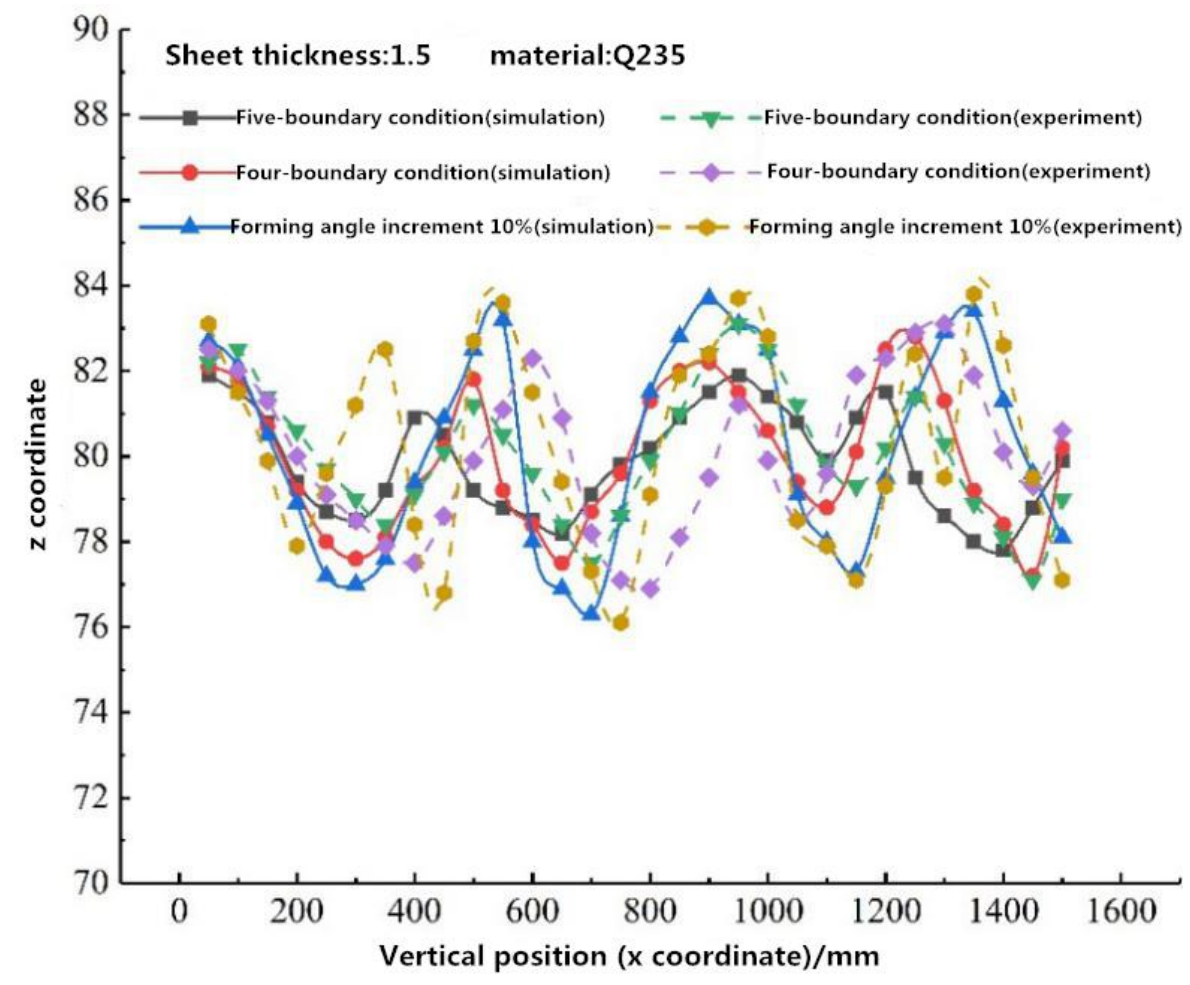

Fig 18 Comparison of experimental and simulated profiles of the flange edge under different forming angle distribution methods

From these results, it is shown that the contour curve and the fluctuation range of the $z$ coordinate under the five-boundary condition mode, whether simulated or experimental, is relatively smooth.

Overall, the trend of the experimentally results is consistent with the simulated data, however the distribution of the simulated data fluctuates more. This difference between experiment and simulation is caused by the frictional forces on the roll, the manufacturing error of the equipment, and the error generated during the sheet feeding.

In order to show the influence of different bending angle distribution methods on the warpage defect, we compare the data collected through simulation and experimental and Equation (7), thereby calculating the warpage fluctuation, $\Delta z$, under different forming angle distribution and the maximum deviation of z-axis coordinate $z^{\prime}$, as shown in Table 7 and Figure 19.

Table 7 Fluctuation of edge wave, $\Delta z$, and maximum deviation, $z^{\prime}$, of the edge under different forming angle distribution methods 


\begin{tabular}{ccccccc}
\hline distribution method & & & & forming angle \\
& simulation & experiment & simulation & experiment & simulation experiment \\
\hline$\Delta z$ & 1.23 & 1.54 & 1.62 & 1.78 & 2.81 & 2.33 \\
\hline$z^{\prime}(\mathrm{mm})$ & 2.09 & 3 & 2.32 & 3.19 & 3.9 & 4.2 \\
\hline
\end{tabular}

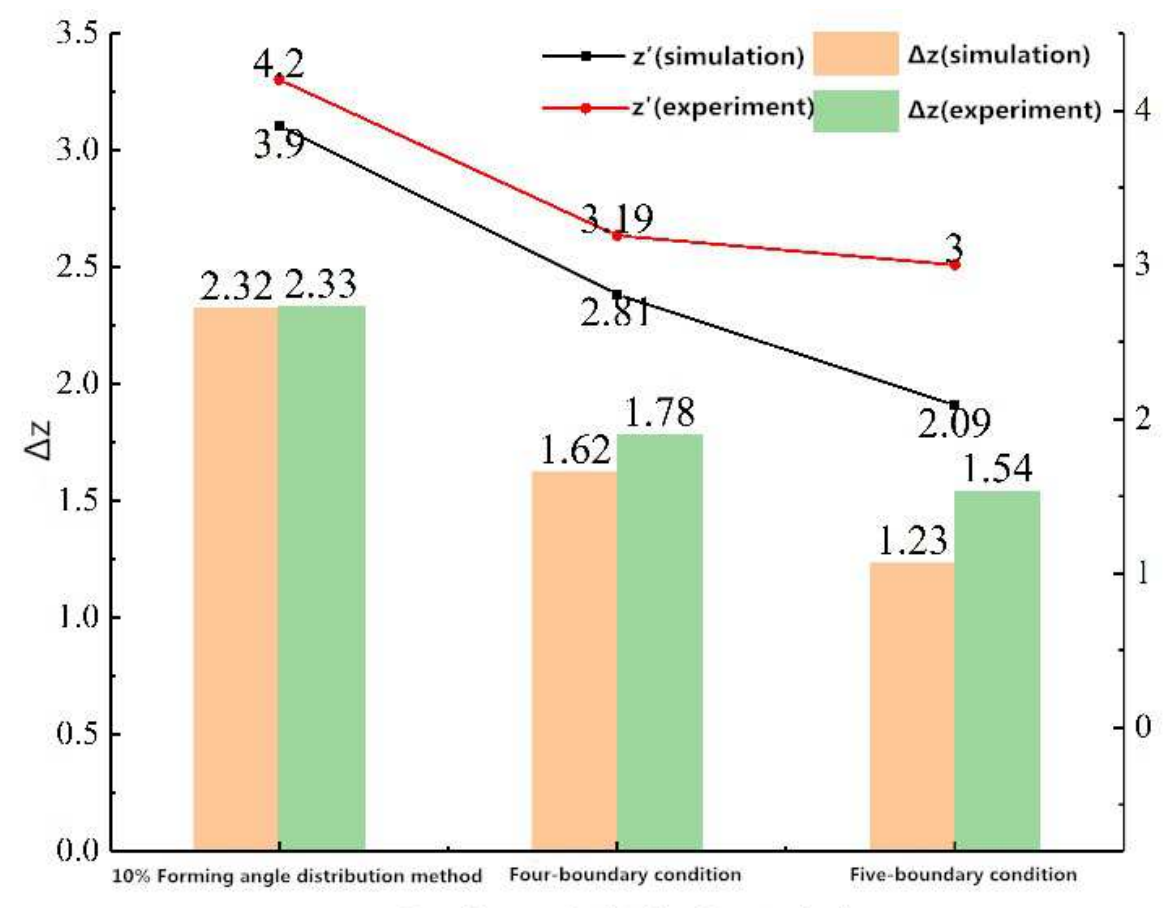

Forming angle distribution method

Fig.19 Comparison of experimental and simulated results of fluctuation of edge wave, $\Delta z$, and maximum deviation, $z^{\prime}$, of the edge under different forming angle distribution methods

Table 7 and Figure 19 show that the warpage fluctuation, $\Delta z$, and maximum deviation, $z^{\prime}$, produced by roll forming of the hat-shaped piece based on the five-boundary condition is smaller than the amount of warpage produced by the other bending angle distribution methods.

Through this comparison of experimental and simulation results, it can also be found that the warpage fluctuation and maximum deviation of the z-axis coordinate calculated from the experimental data are slightly larger than the simulated value. This result is due to the unavoidable errors in actual production. However, the trend remains consistent with the simulation results.

3.3 The influence of different sheet thicknesses on simulated and experimental results of warpage defects of hat-shaped parts 
Based on the optimized five-boundary condition forming angle distribution method, the influence of sheet thickness $(1.0 \mathrm{~mm}, 1.5 \mathrm{~mm}$, and $2.0 \mathrm{~mm})$ on the warpage defects is evaluated. The material used in this study was also Q235 steel.

Once simulation and experiment were complete, the coordinates of each node $(\mathrm{x}, \mathrm{z})$ on the edge were measured and recorded. The contour curves of the longitudinal nodes are shown in Figure 20.

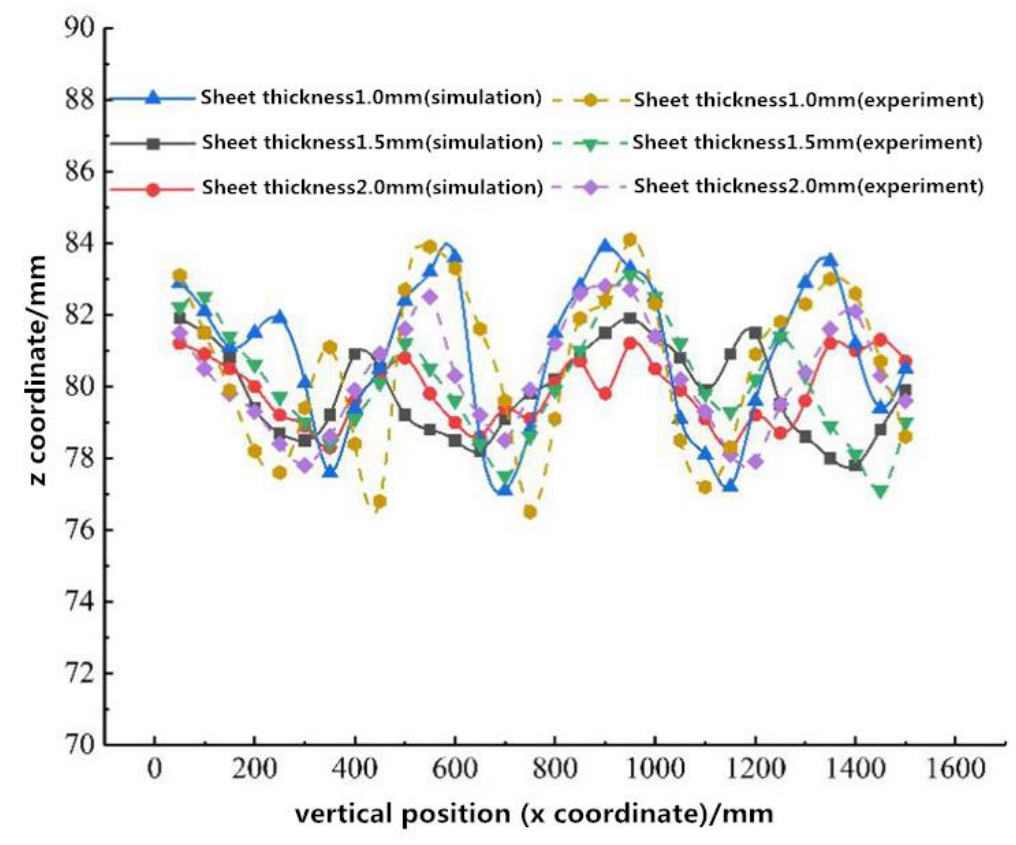

Fig.20 Comparison of resulting experimental and simulated profiles under different sheet thicknesses

In order to show the influence of sheet thickness on the warpage defect, the data collected by simulation experiment, and Equation (11) are show in in Table 8 and Figure 21 with their calculated warpage fluctuation, $\Delta z$, and maximum deviation of $z$-axis coordinate, $z^{\prime}$, for different forming angle distributions.

Table 8 Fluctuation, $\Delta z$, of edge wave and maximum deviation, $z^{\prime}$, of the edge under different sheet thicknesses

1.0

1.5

2.0

Sheet thickness $(\mathrm{mm})$

simulation experiment simulation experiment simulation experiment

\begin{tabular}{lllllll}
\hline$\Delta z$ & 2.01 & 2.21 & 1.23 & 1.54 & 0.91 & 1.46
\end{tabular}




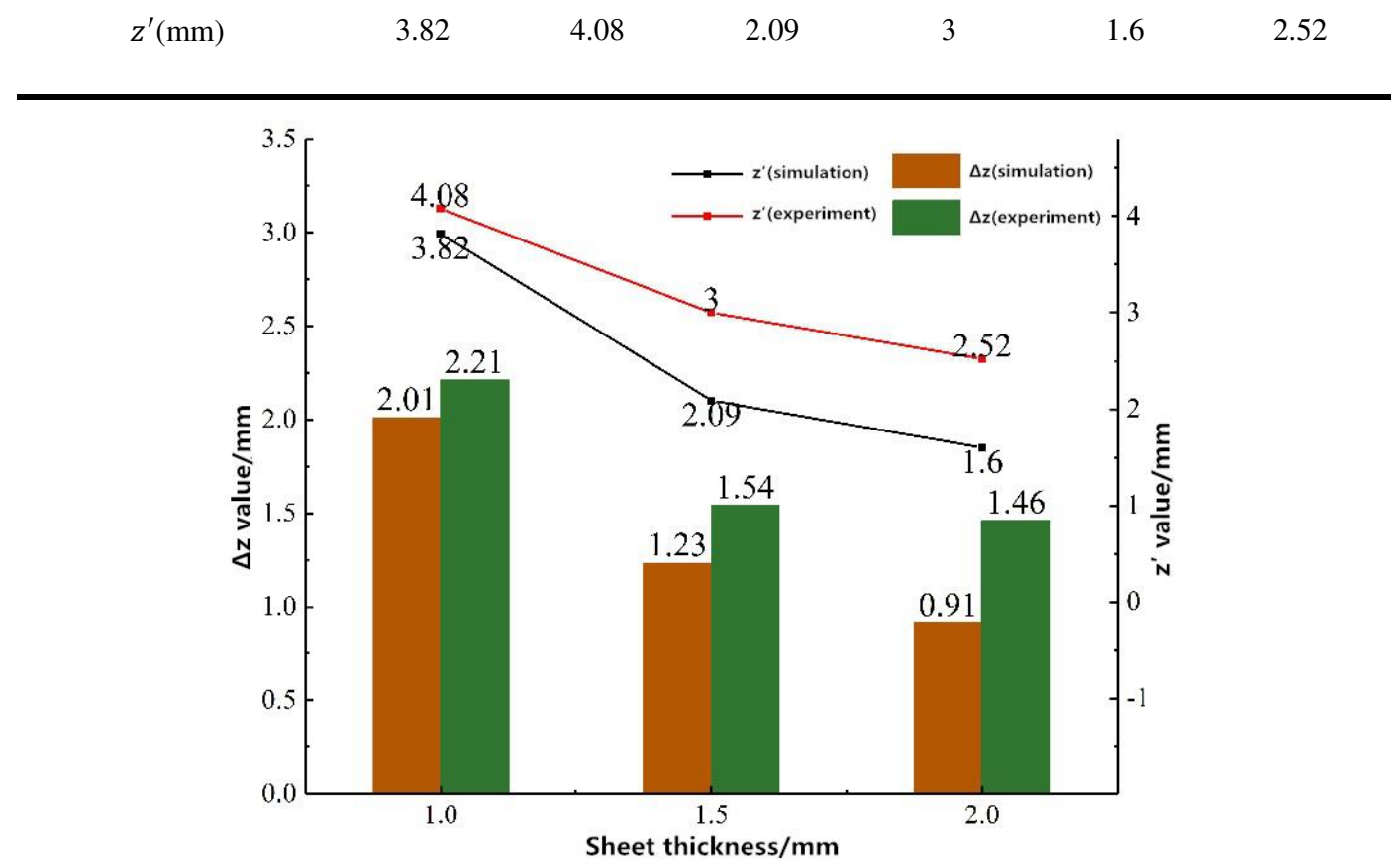

Fig. 21 Comparison of experiment and simulation of fluctuation, $\Delta z$, and maximum deviation, $z^{\prime}$, of the flange edge under different sheet thicknesses

Table 8 and Figure 21 show that when the plate thickness increases, the warpage fluctuation, $\Delta z$, and the maximum deviation of the $z$-axis coordinate are significantly reduced; that is, the warpage fluctuation of the flange edge decreases with the increase of the plate thickness. The thicker the sheet, the less likely it is to warp. These experimental results are in good agreement with simulation. However, while increasing the thickness can help reduce the degree of warpage, it may also introduce defects such as springback or aggravation, thus in actual production, the thickness of the selected sheet should be chosen appropriately.

\subsection{Influence of yield strength on the simulation and experimental results of warpage defects of hat-shaped parts}

This section focuses on the five-boundary conditions of the forming angle distribution method when the sheet thickness is $1.5 \mathrm{~mm}$. Select DP780 steel $\left(\sigma_{s}=578 \mathrm{MPa}\right)$ is compared to Q235 steel $\left(\sigma_{s}=235 \mathrm{MPa}\right)$ to study the effect of material yield strength on the warpage defect.

After the simulation and experiment were finished, we collected and measured the $x$ and $z$ coordinates of each node at the edge of the hat-shaped piece and record the values. These values are compared in Figure 22. 


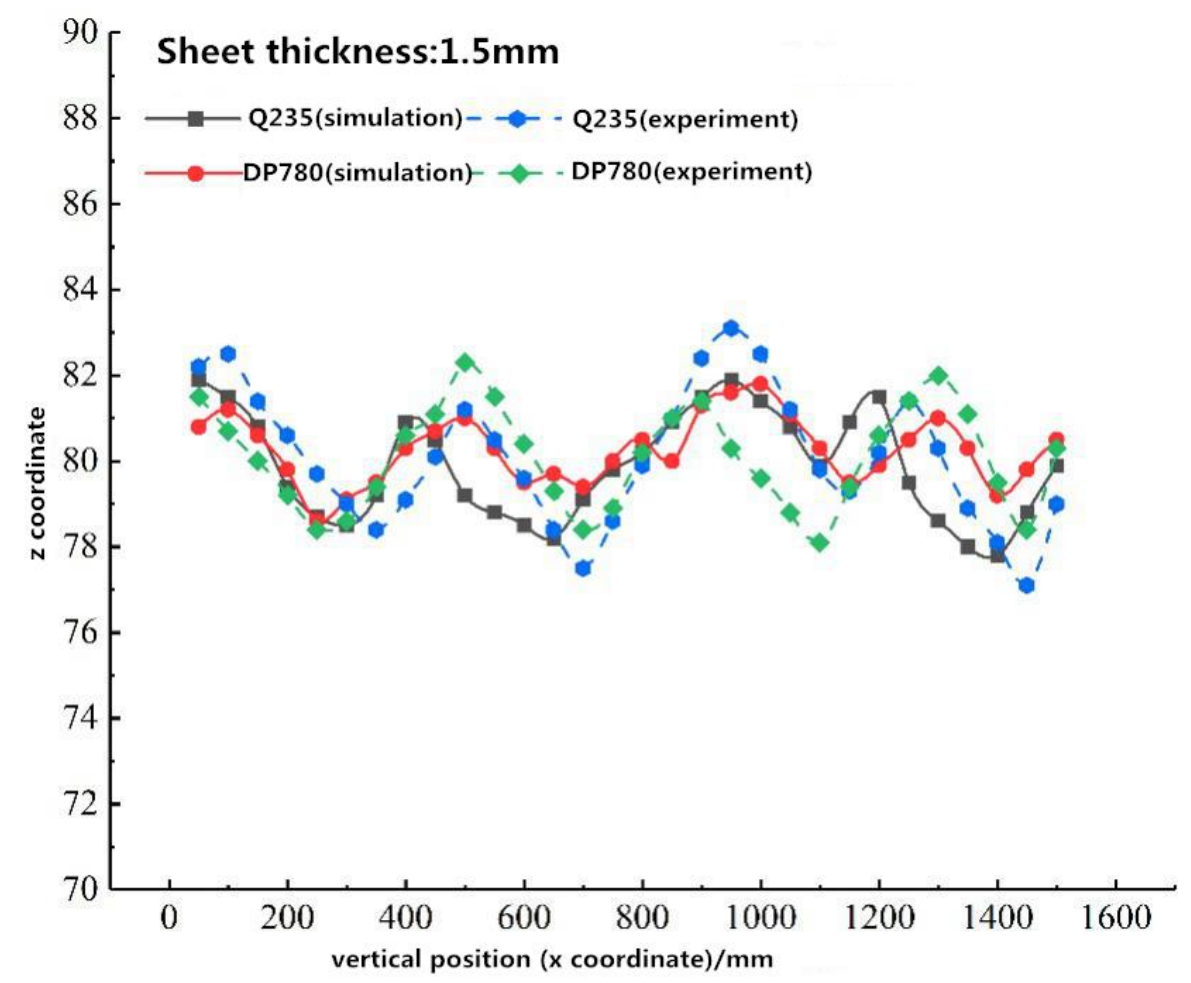

Fig.22 Comparison of curves From experimental and simulated profiles of the flange edge under different sheet yield strengths

Excluding the small data differences caused by strong force factors, the trend of the experimentally measured data is consistent with the simulated data. The greater the yield strength of the material, the smaller the amount of warpage.

Calculations of the warpage fluctuation, $\Delta z$, under different forming angle distributions and the maximum deviation of $z$-axis coordinate, $z^{\prime}$, are shown in Table 9 and Figure 23.

Table 9 Fluctuation, $\Delta z$, of edge wave and maximum deviation, $z^{\prime}$, of the flange edge under different sheet yield strengths

\begin{tabular}{ccccc}
\hline & \multicolumn{2}{c}{ Q235 } & \multicolumn{2}{c}{ DP780 } \\
material & simulation & experiment & simulation & experiment \\
& 1.23 & 1.23 & 0.76 & 0.76 \\
\hline$\Delta \mathrm{z}$ & 2.09 & 2.09 & 1.66 & 1.66 \\
\hline$z^{\prime}(\mathrm{mm})$ & & & & \\
\hline
\end{tabular}




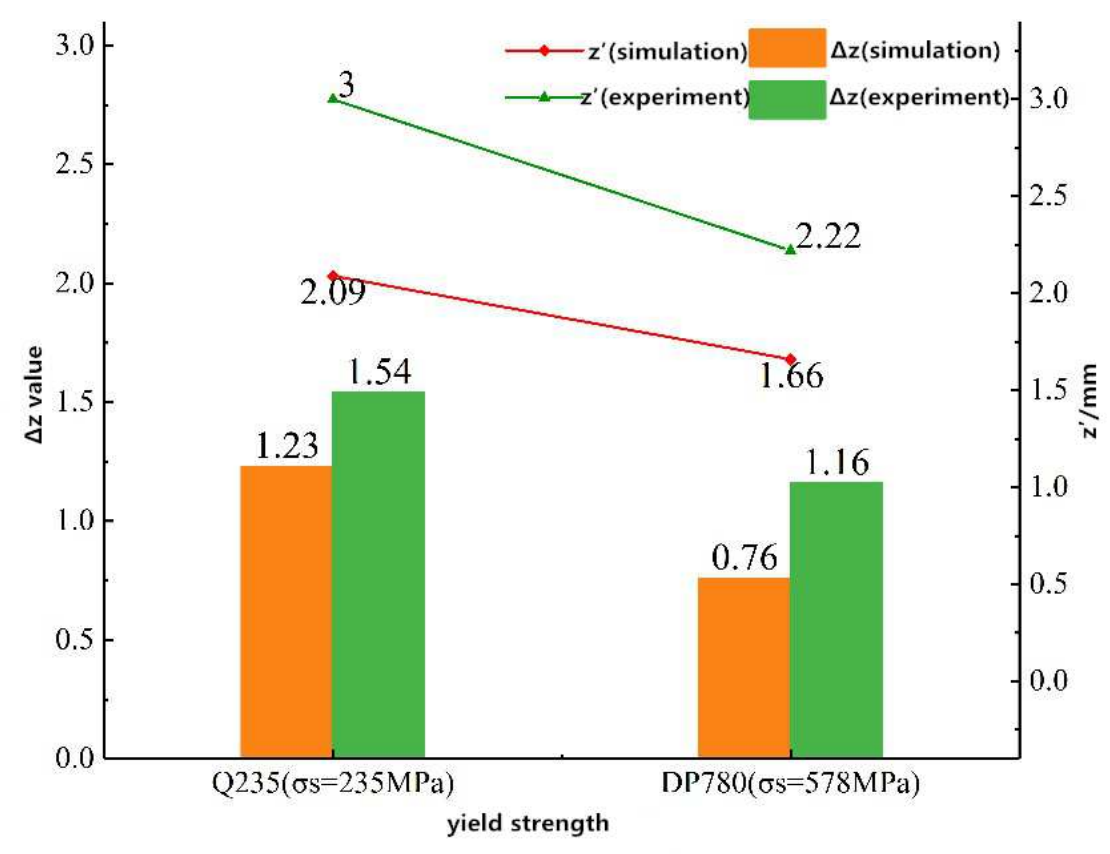

Fig.23 Comparison of experiment and simulation of fluctuation of edge wave, $\Delta z$, and maximum deviation, $z^{\prime}$, of the flange edge under different sheet yield strengths

Table 9 and Figure 23 show the the warpage fluctuations in DP780 and Q235 to be 0.76 and 1.23 in simulation, respectively, and that the maximum deviation of the $\mathrm{z}$-axis coordinate is 1.66 $\mathrm{mm}$ and $2.09 \mathrm{~mm}$. However, the warpage fluctuations, $\Delta z$, as calculated from the experimental data, are 1.16 and 1.54, while the maximum deviations of the $z$-coordinates are $2.22 \mathrm{~mm}$ and $3 \mathrm{~mm}$. Thus, we see that the yield strength of the material has a great influence on warping defects and that the fluctuation of the flange edge decreases with increased yield strength. However, the forming quality of sheet materials with higher yield strength is not as good and as discussed in the previous section, the cap forming parts will have greater springback.

\section{Conclusion}

This manuscript examines the cap-shaped roll bending part of a small section profile that is common in industrial production. We proposed and optimized a five-boundary condition forming angle distribution function. For the first time, the forming angle, sheet thickness, and sheet yield strength were analyzed by ABAQUS finite element simulation study under the proposed forming angle distribution method. Three forming parameters were shown to impact warpage defects and roll forming experiments were completed and compared to simulations. The conclusions are as follows: 
(1) By using the ABAQUS software to simulate the stress and strain of the hat-shaped piece after roll forming, we found that the warping defect of the flange is caused by the uneven distribution of longitudinal stress and longitudinal plastic strain on the flange of the hat.

(2) Through use of the forming angle on the three distribution modes, the simulation of the flange edge warpage fluctuation, $\Delta z$, and the maximum deviation of $z$-coordinates were be obtained. The contour curve of the flange edge of the hat-shaped piece under the five-boundary condition angle distribution method is relatively smooth. This result is consistent with experiment. Thus, the amount of warpage under the five-boundary condition bending angle distribution method is less than the degree of warpage produced by the other two bending angle distribution methods.

(3) The influence of different forming parameters on warpage was studied on the basis of the distribution method of the five boundary condition. The experimentally collected data is consistent with the law of the warpage of the cap flange edge as obtained by numerical simulation. That is, the amount of warpage fluctuation decreases with increasing plate thickness.

(4) When switching the material to DP780, we found the contour curve of the flange edge of the hat-shaped piece to be relatively gentle. These experimental results are consistent with the influence law obtained by the numerical simulation. That is, the amount of warpage fluctuation of the flange edge decreases as the yield strength of the sheet increases.

\section{References}

[1] HIROSHI O, TAKASHI J, HARUO K, et al. Computer-Aided Design for Cold Roll Forming of LightGauge Steel Members[J]. JSME international journal. Ser. 1, Solid mechanics, strength of materials, 1990, 33(2): 220-226.

[2] BIDABADI B S, NAEINI H M, TAFTI R A, et al. Experimental investigation of the ovality of holes on pre-notched channel products in the cold roll forming process[J]. Journal of Materials Processing Technology, 2015, 225: 213-220.

[3] SHIRANI BIDABADI B, MOSLEMI NAEINI H, et al. Experimental study of bowing defects in prenotched channel section products in the cold roll forming process[J]. The International Journal of Advanced Manufacturing Technology, 2016, 87(1-4): 997-1011.

[4] HAN Z W, LIU C, LU W P, et al. The effects of forming parameters in the roll-forming of a channel section with an outer edge[J]. Journal of Materials Processing Tech, 2001, 116(2): 205-210. 
[5] M. Salmani Tehrani, P. Hartley, H. Moslemi Naeini, H. Khademizadeh. Localised edge buckling in cold roll-forming of symmetric channel section[J]. Thin-Walled Structures 2006,44: 184-196.

[6] 罗晓亮, 曾国, 李淑慧, 等. 材料参数对高强钢辊弯成形边波影响的有限元分析 $[\mathrm{J}]$. 上海交通大学学 报. 2008, 42(5): 744-747.

[7] 王镇晓, 赵亦希, 孔庆帅, 于忠奇. 柔性滚弯成形工艺中边波的控制方法 $[\mathrm{J}]$. 上海交通大学学 报, 2014, 48(05)：624-628+635.

[8]＼cjkstart黎宇. 薄壁槽钢零件辊弯成形数值模拟及其边波缺陷研究 [D]. 湘潭：湘潭大学, 2015.

[9] SU C J, YANG L Y, LOU S M, et al. Optimized bending angle distribution function of contour plate roll forming[J]. The International Journal of Advanced Manufacturing Technology, 2018, 97: 1787-1799

[10] SU C J, YANG L Y, LOU S M, et al. Research on roll forming process based on five-boundary condition forming angle distribution function[J]. The International Journal of Advanced Manufacturing Technology, 2019, 102: 3767-3779. 
Figures

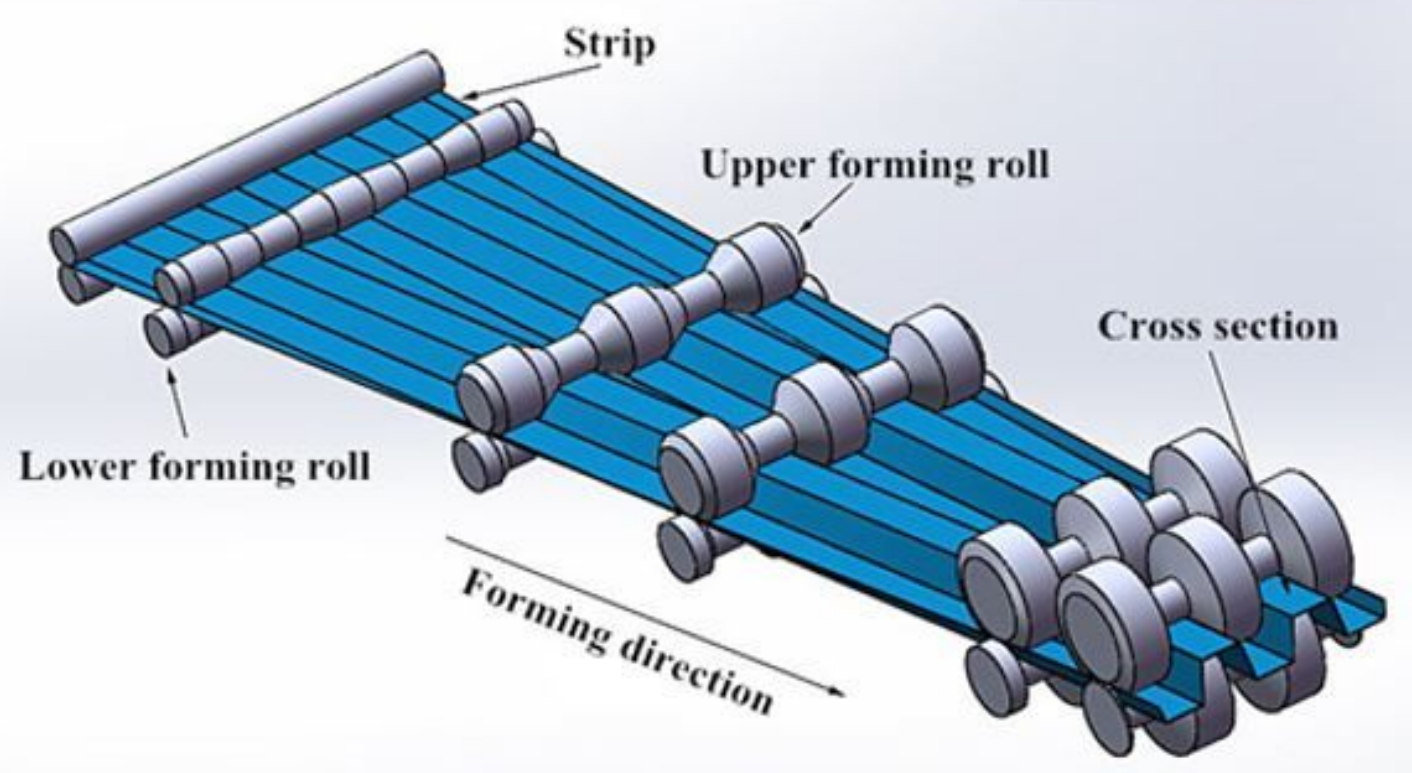

Figure 1

Roll forming process

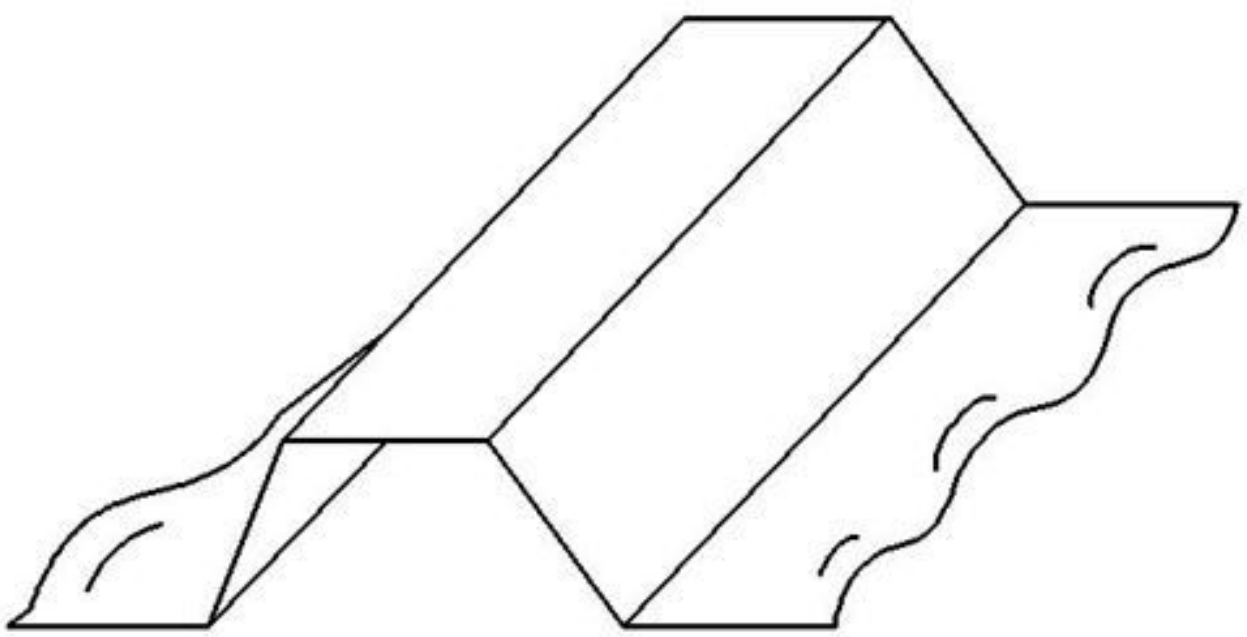


Figure 2

Schematic diagram of edge wave as showing a hat-shaped defect

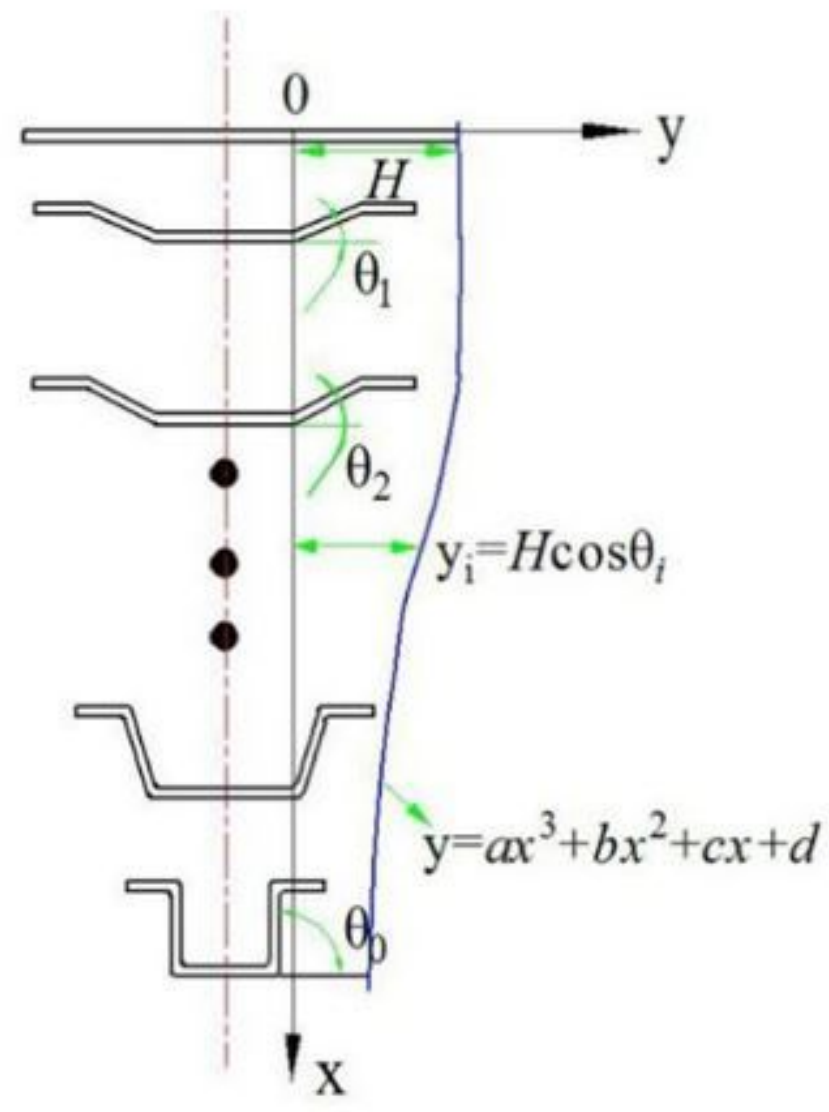

Figure 3

The ideal projection track of the profile edge section in the horizontal plane follows a cubic curve. 


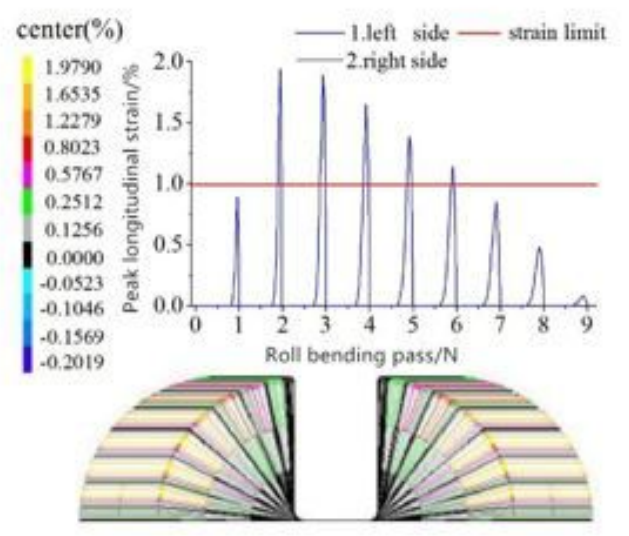

(a) $30 \% \times \theta_{0}$

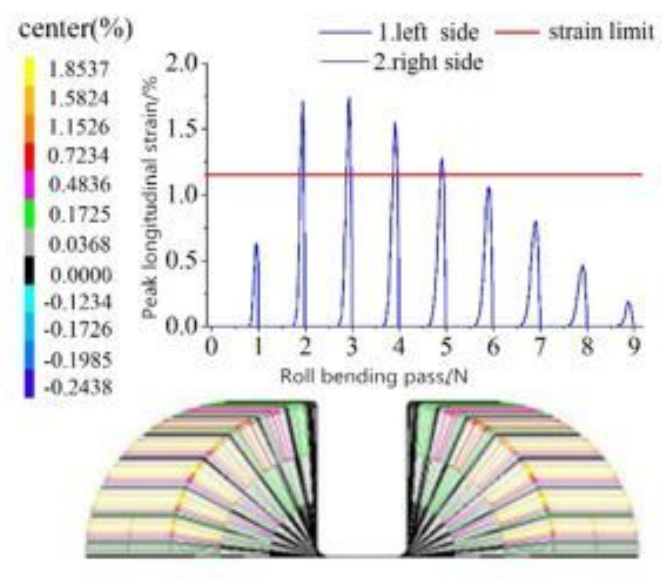

(c) $32 \% \times \theta_{0}$

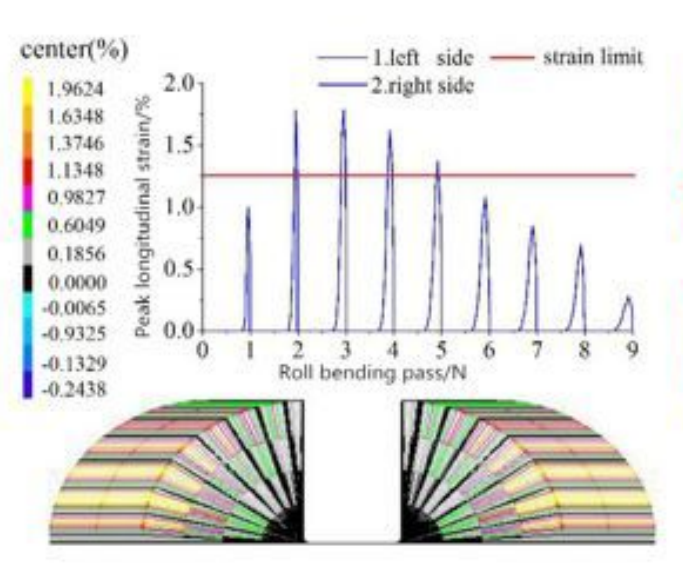

(e) $34 \% \times \theta_{0}$

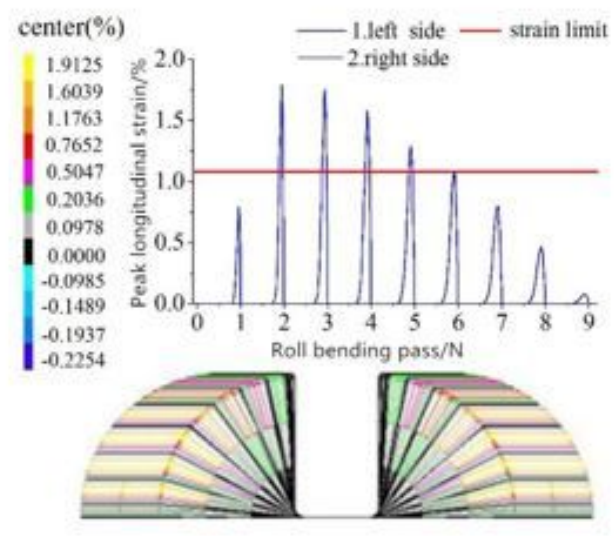

(b) $31 \% \times \theta_{0}$

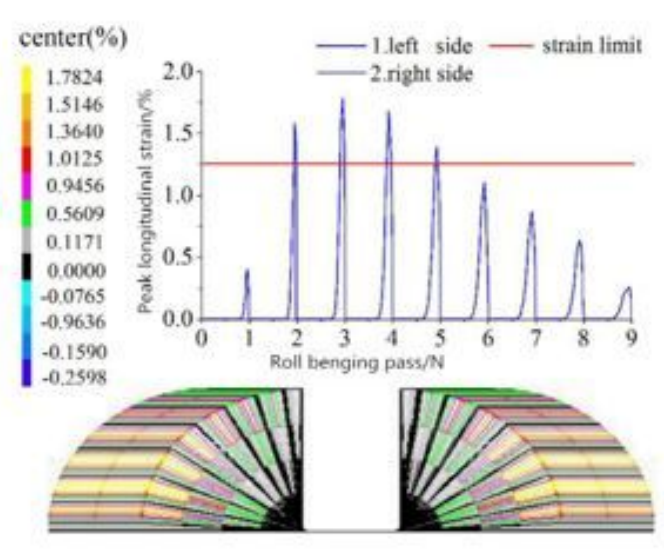

(d) $33 \% \times \theta_{0}$

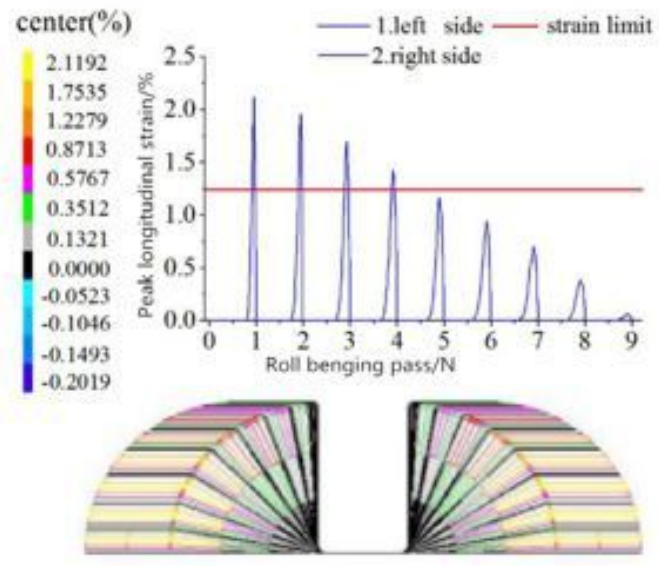

(f) $35 \% \times \theta_{0}$

\section{Figure 4}

Simulation of peak longitudinal strain using five-boundary condition method 


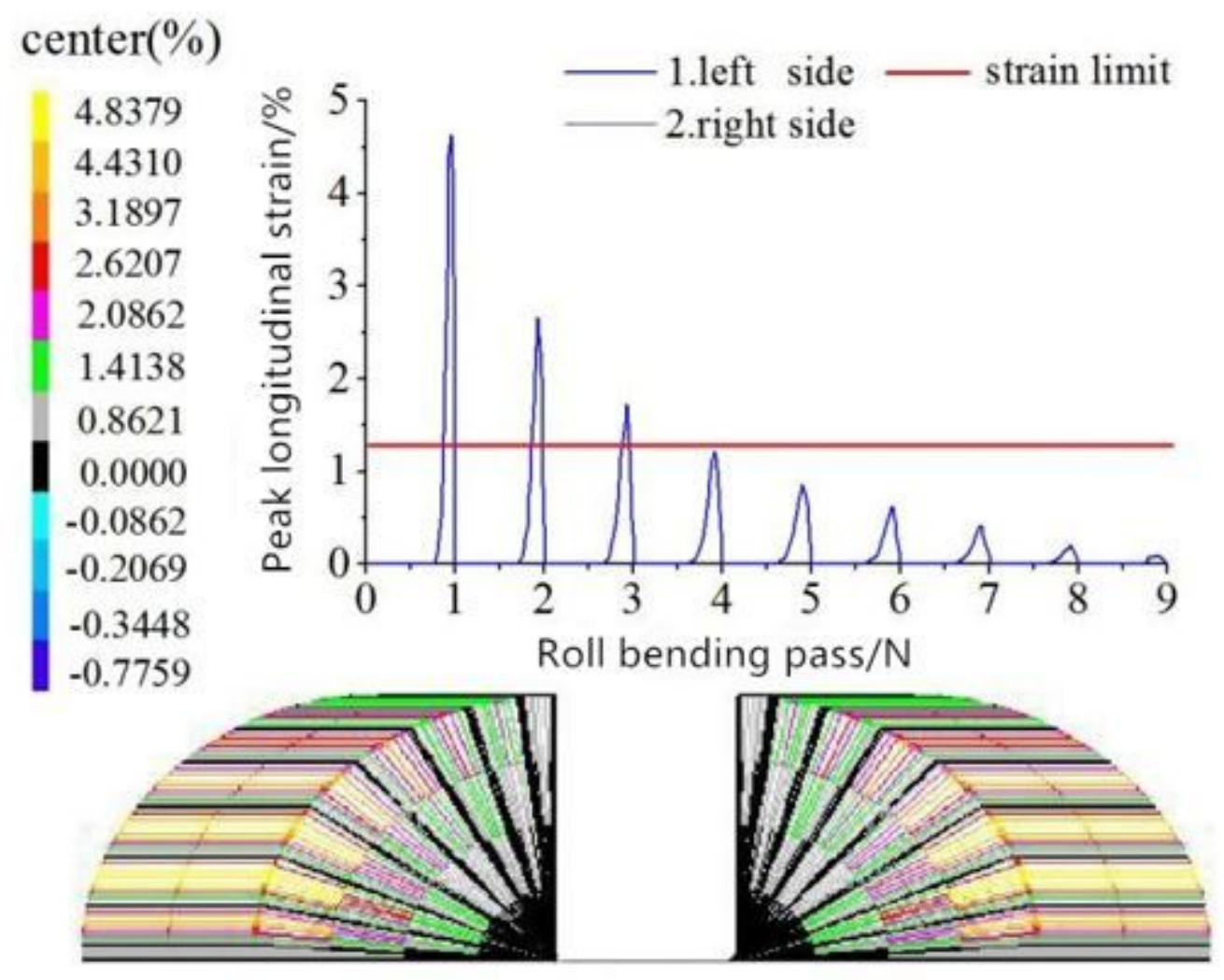

Figure 5

Simulation of peak longitudinal strain based on four-boundary condition 


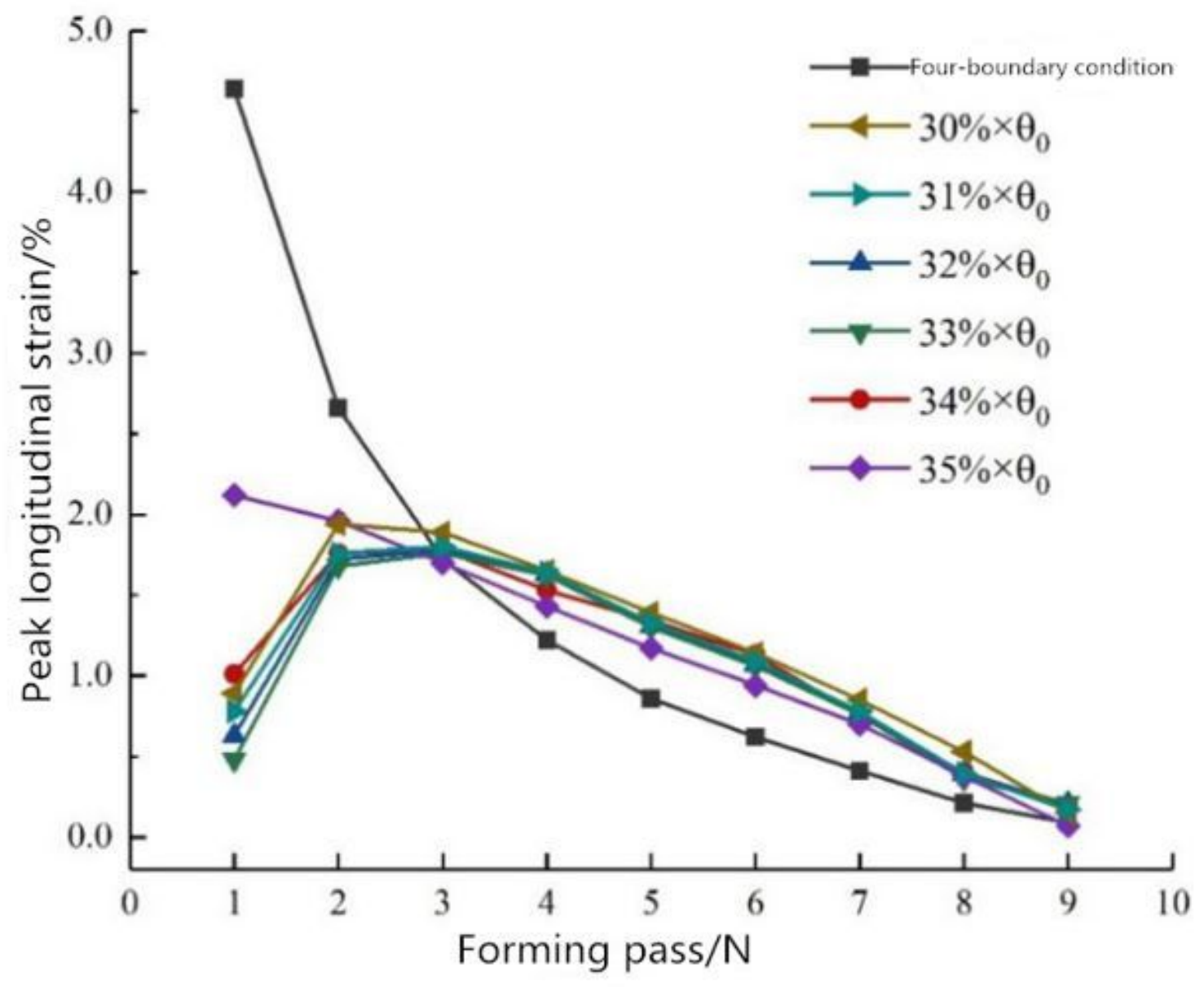

Figure 6

Comparison of peak longitudinal strain between passes based on different boundary conditions 


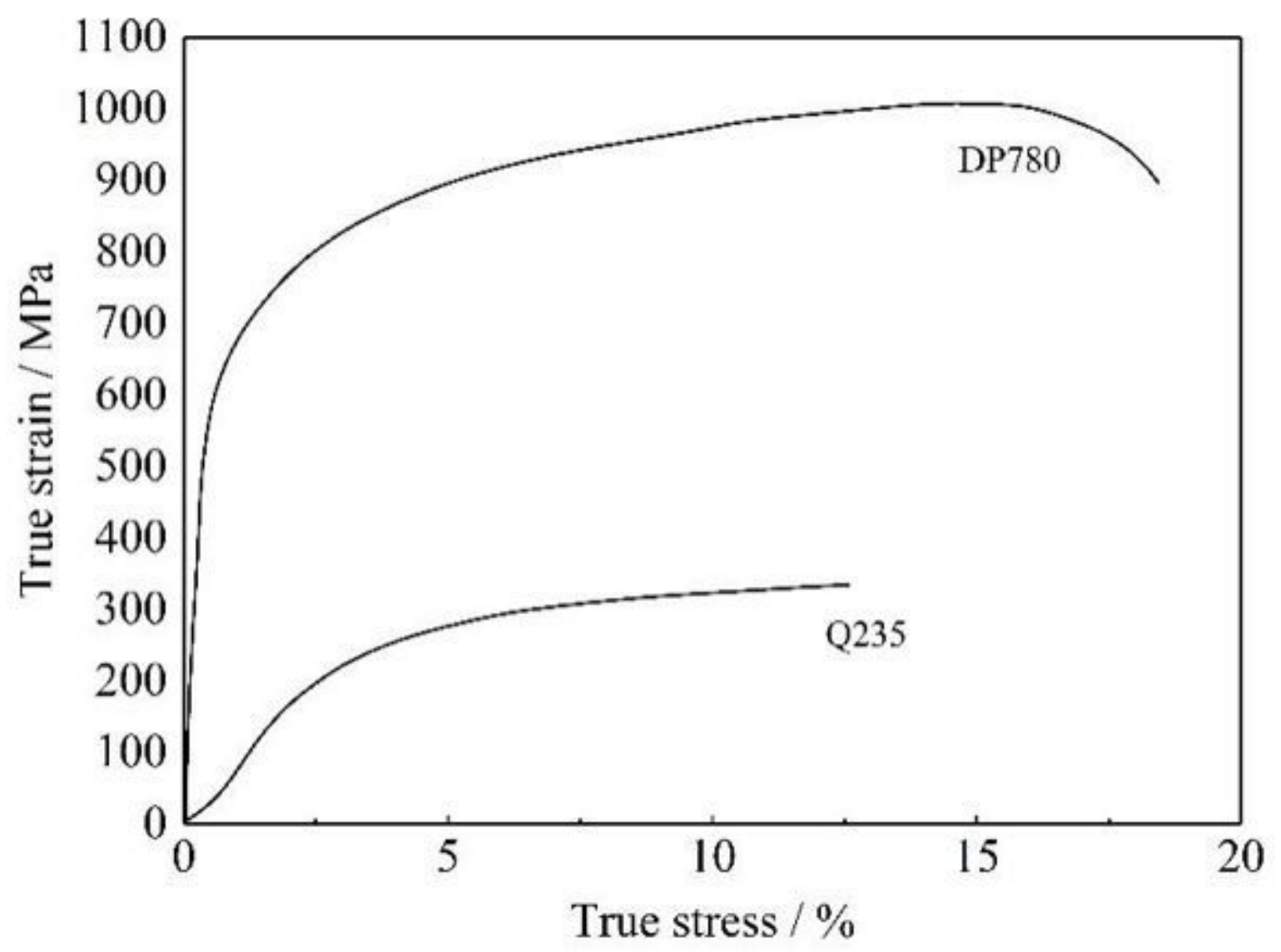

Figure 7

True stress-strain curve

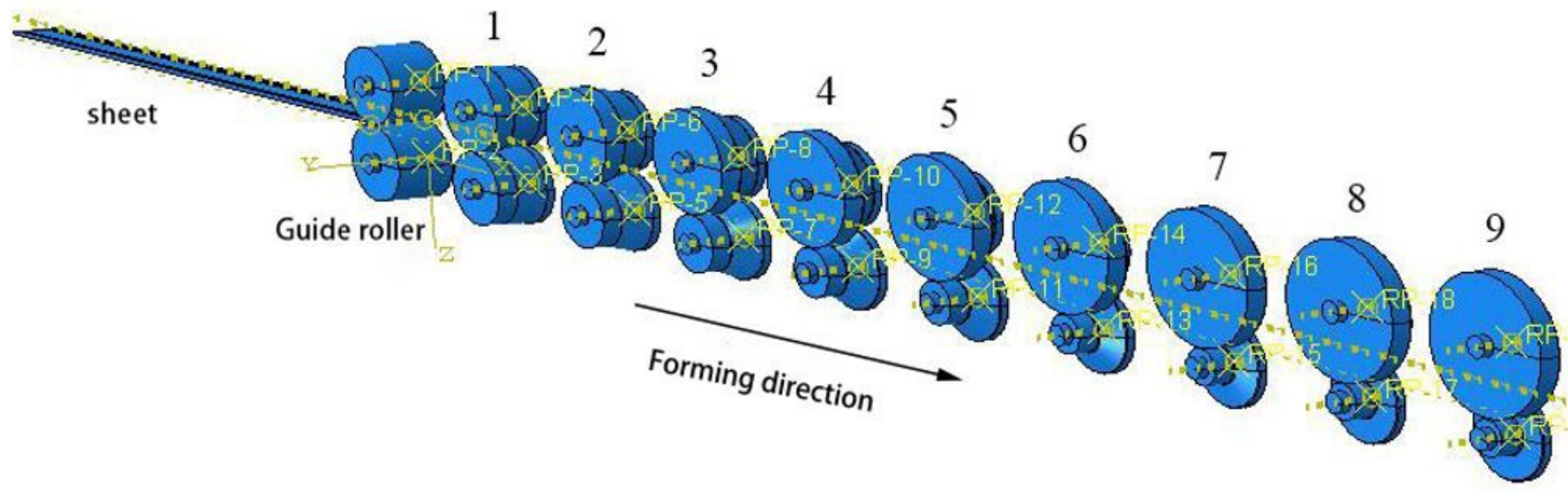

Figure 8

The finite element model of roll forming 


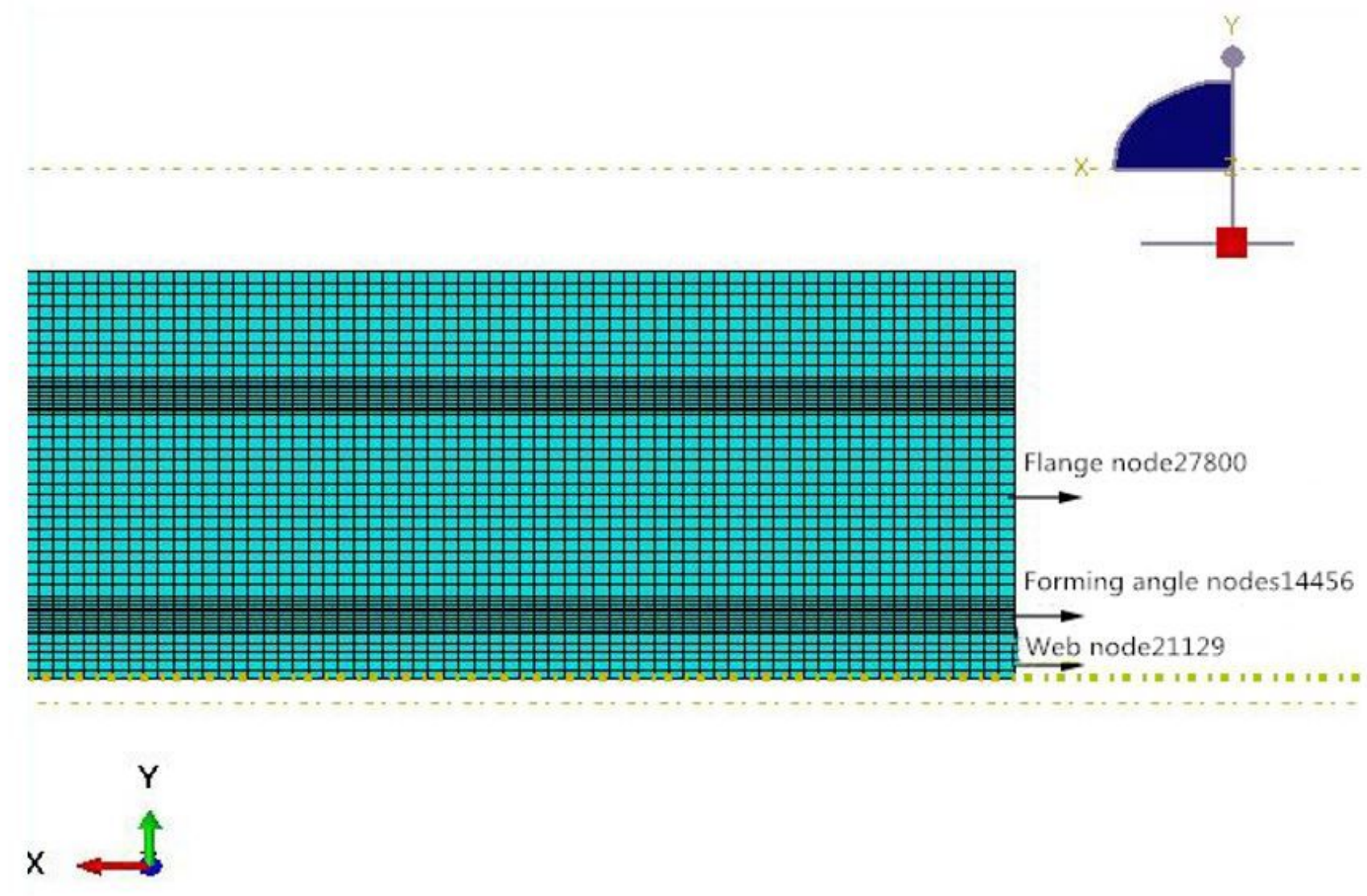

\section{Figure 9}

the grid of the sheet 


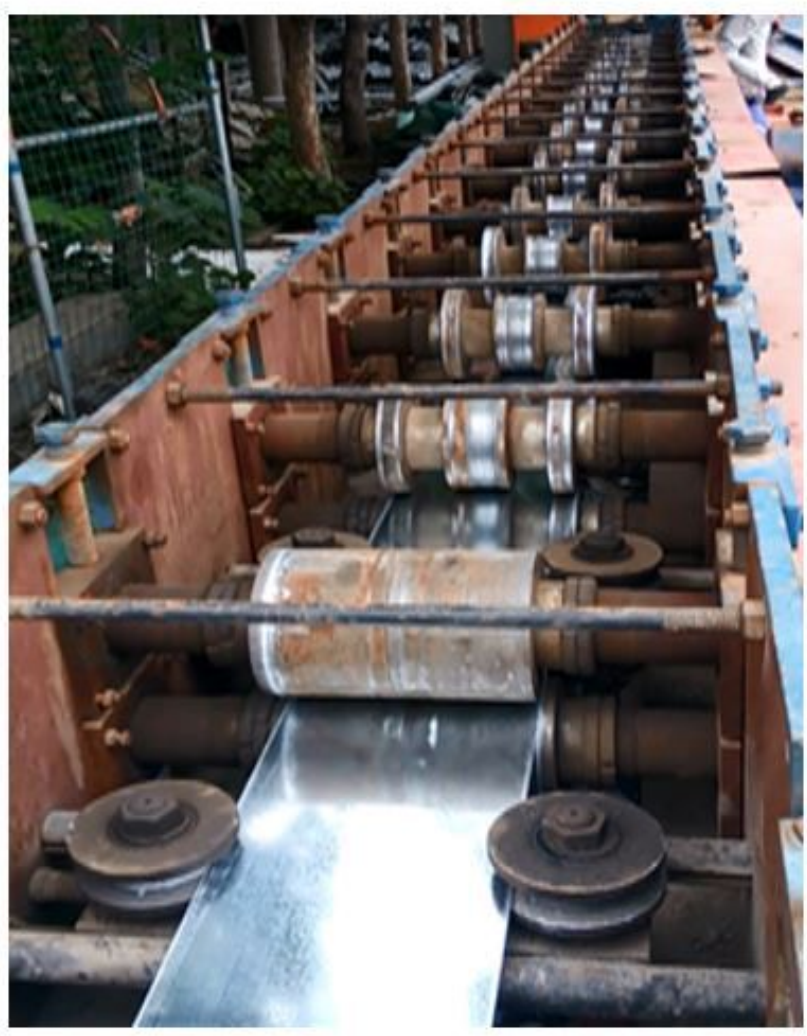

(a) Feed direction

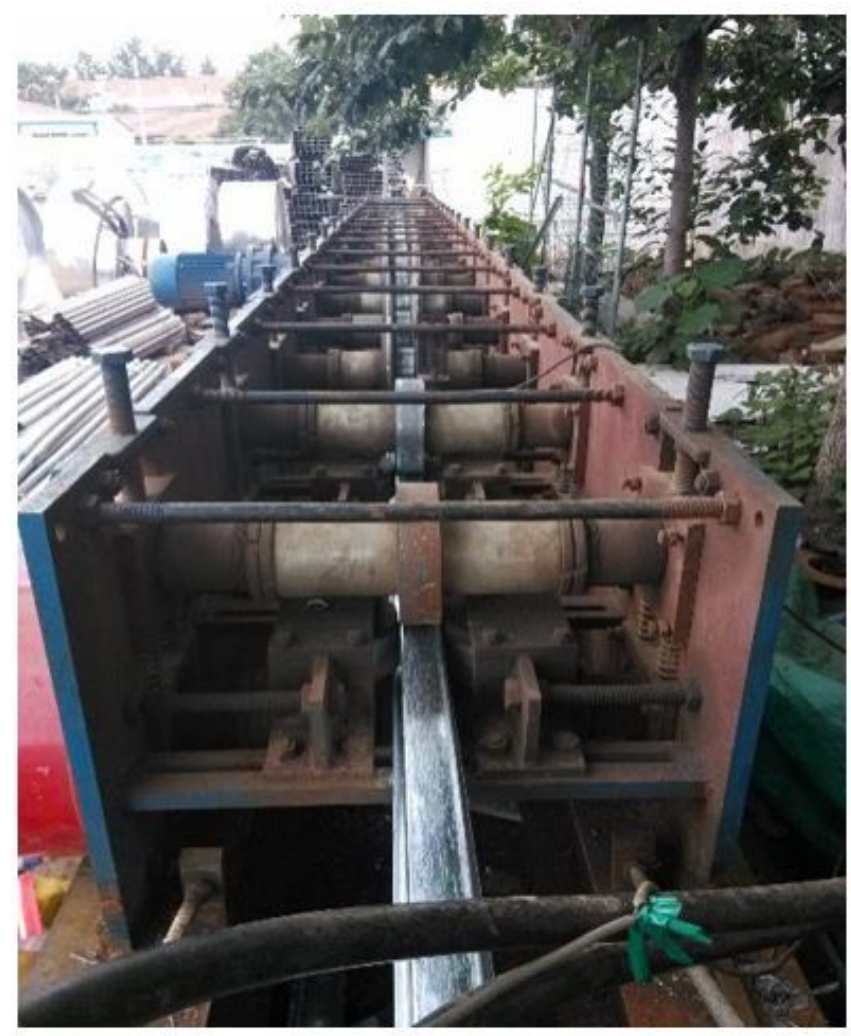

(b) Forming direction

Figure 10

The experimental equipment 


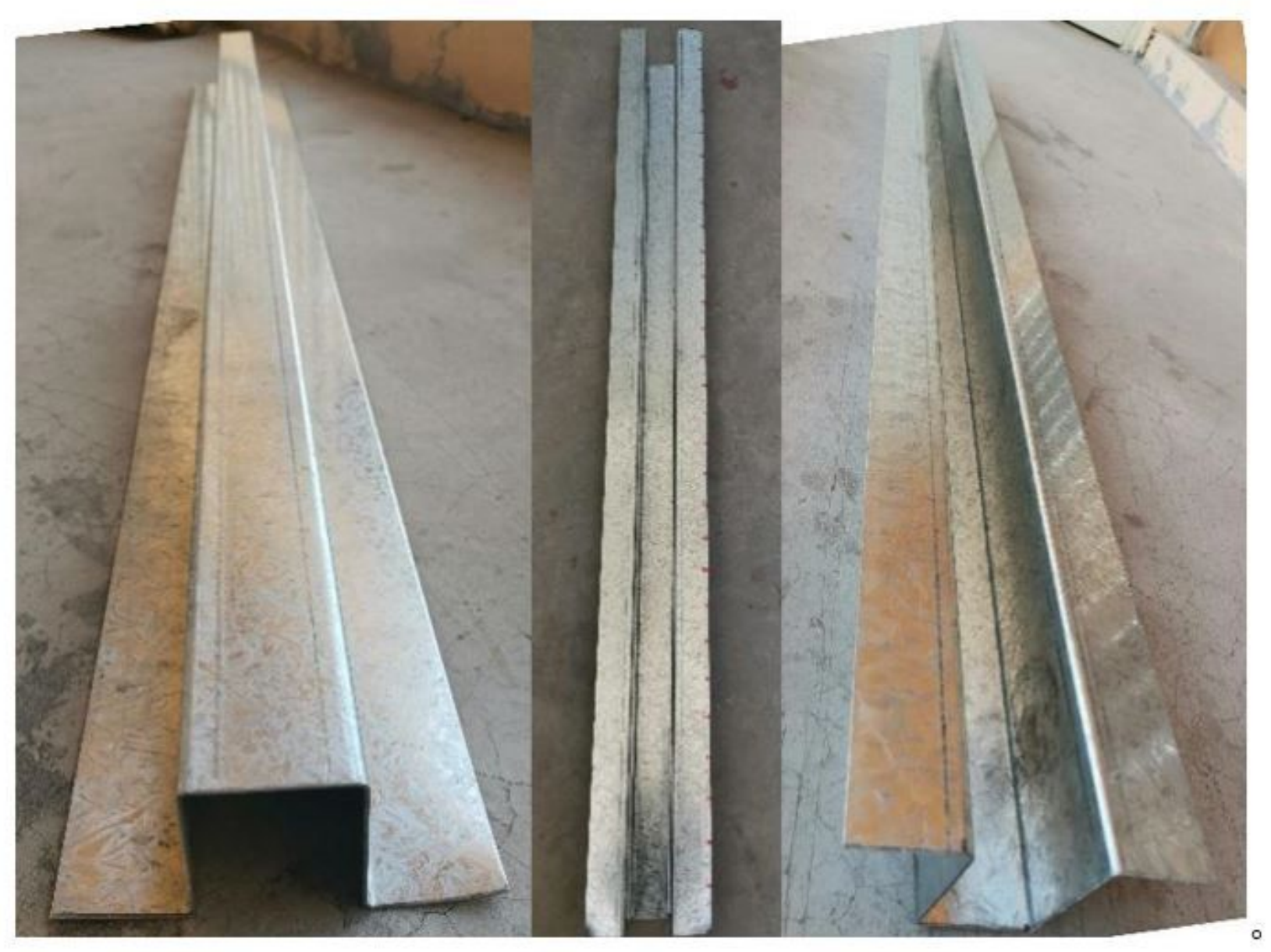

Figure 11

Roll-formed hat-shaped 

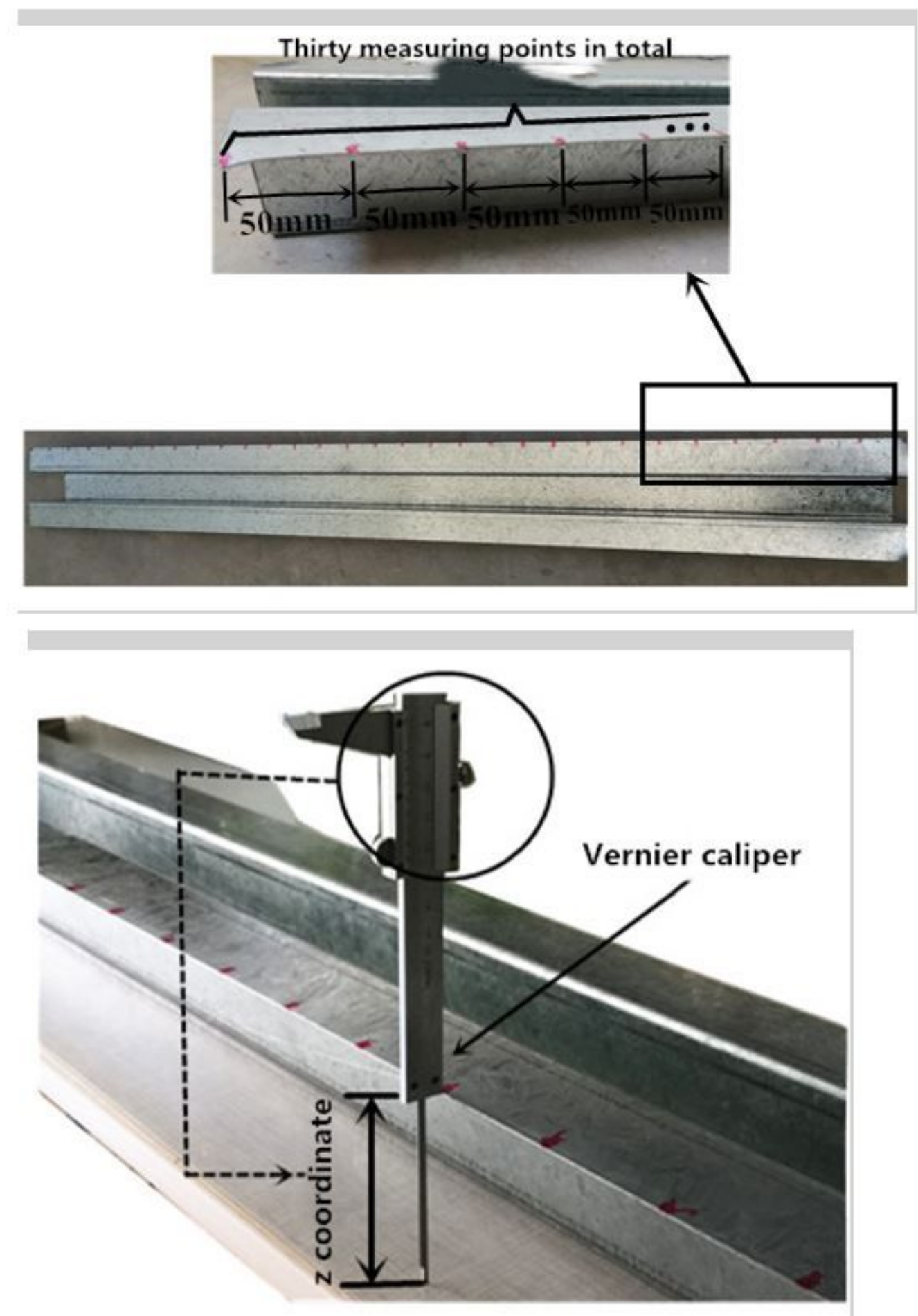

Figure 12

Measuring point of roll-formed hat-shaped and measurement method 


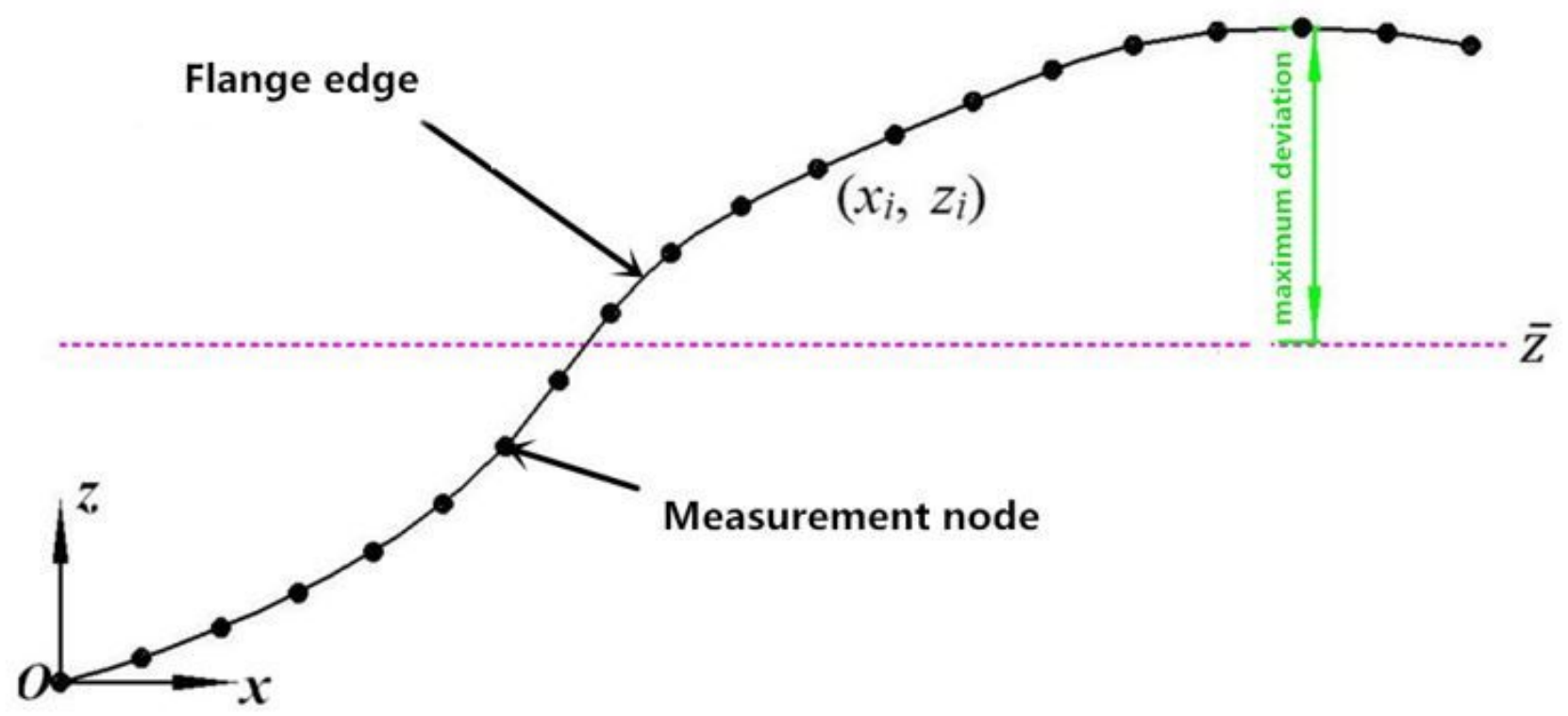

Figure 13

Schematic for evaluating edge wave 


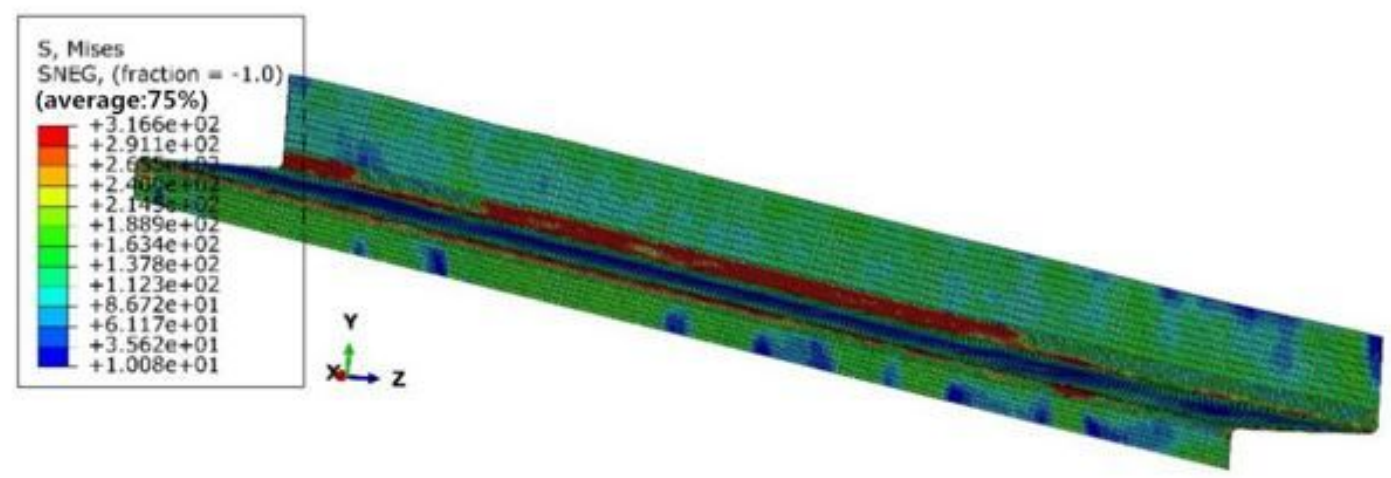

(a) Mises equivalent stress

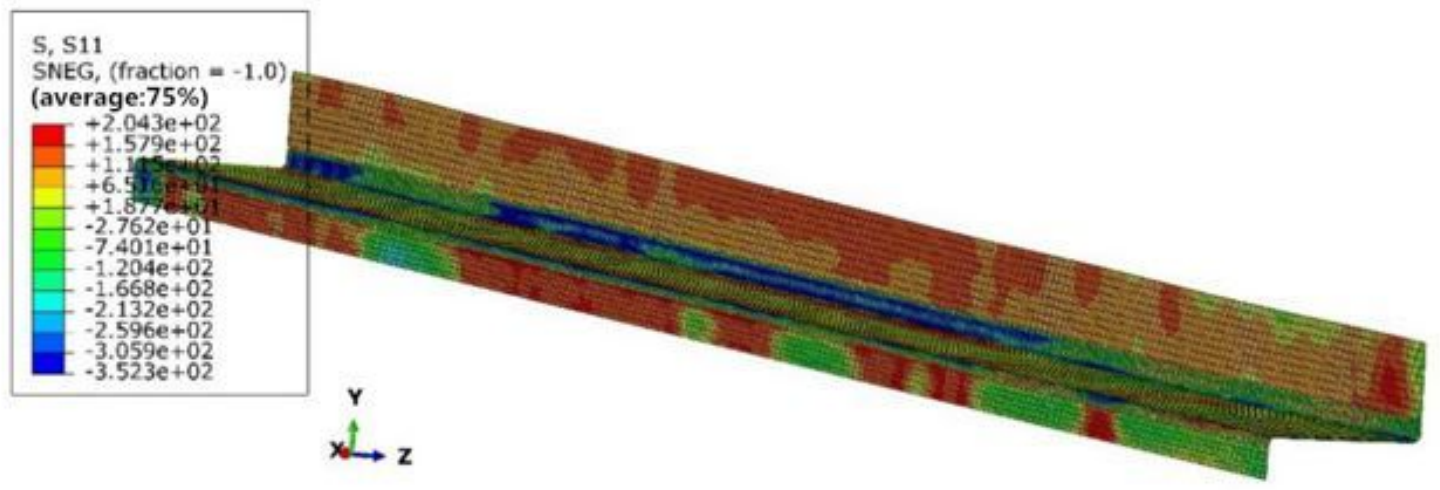

(b) Longitudinal stress, S11

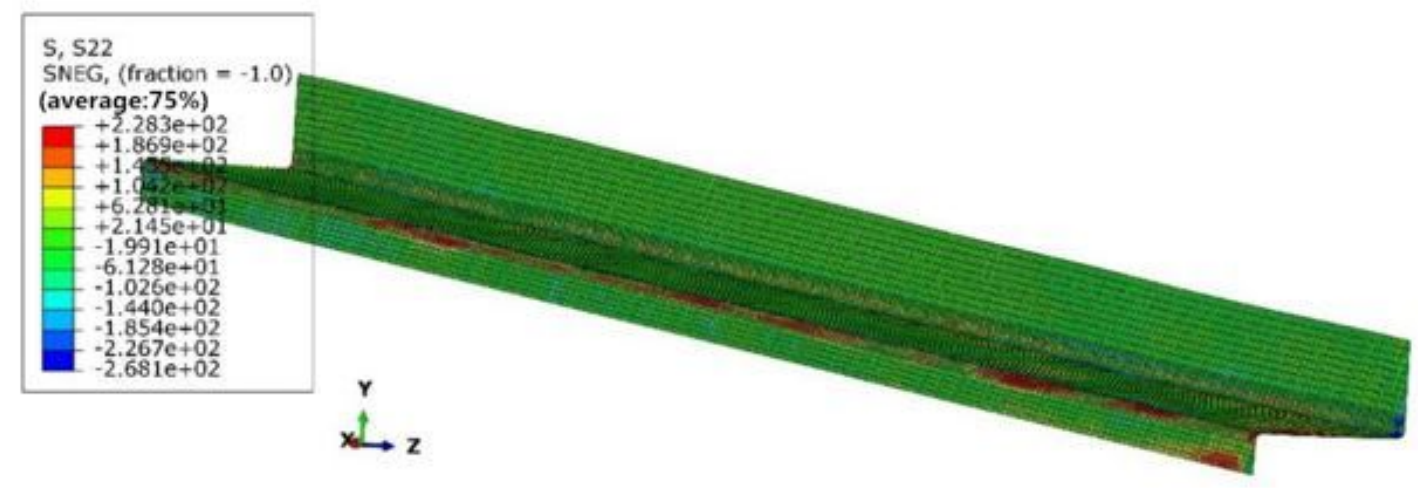

(c) Transverse stress, S22

Figure 14

Stress contour of hat-shaped piece 


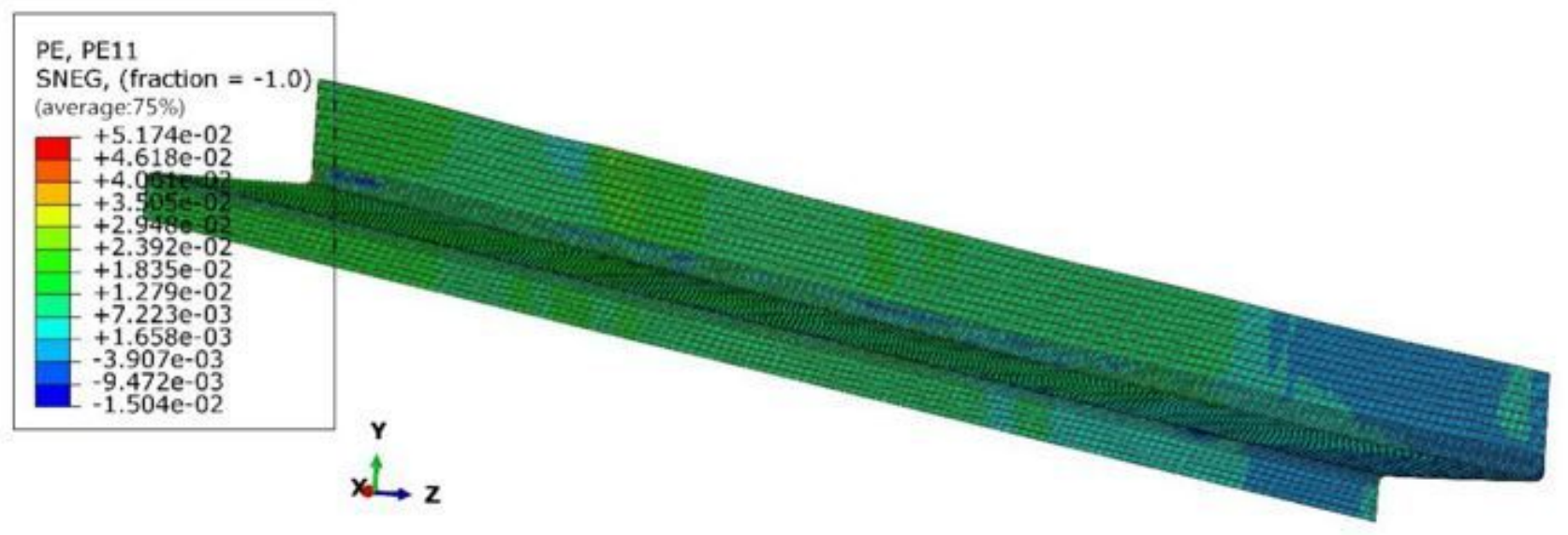

(a) Longitudinal strain, PE11

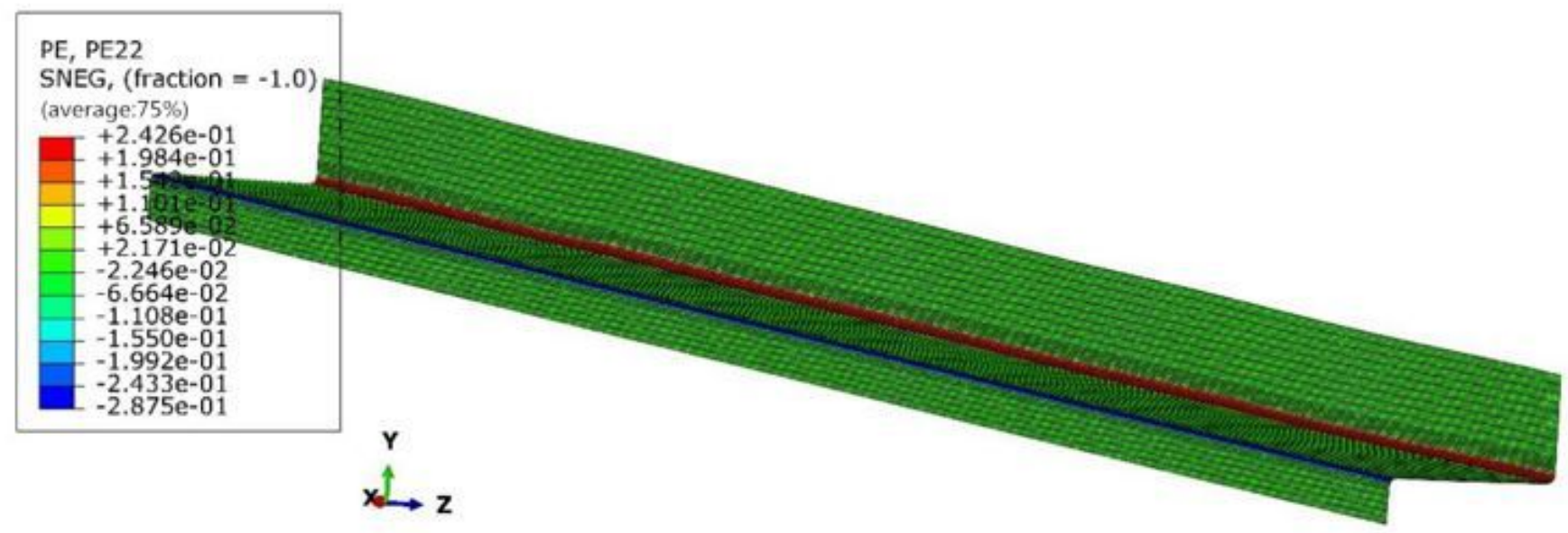

(b) Lateral strain, PE22

\section{Figure 15}

Plastic strain contour of hat-shaped piece 


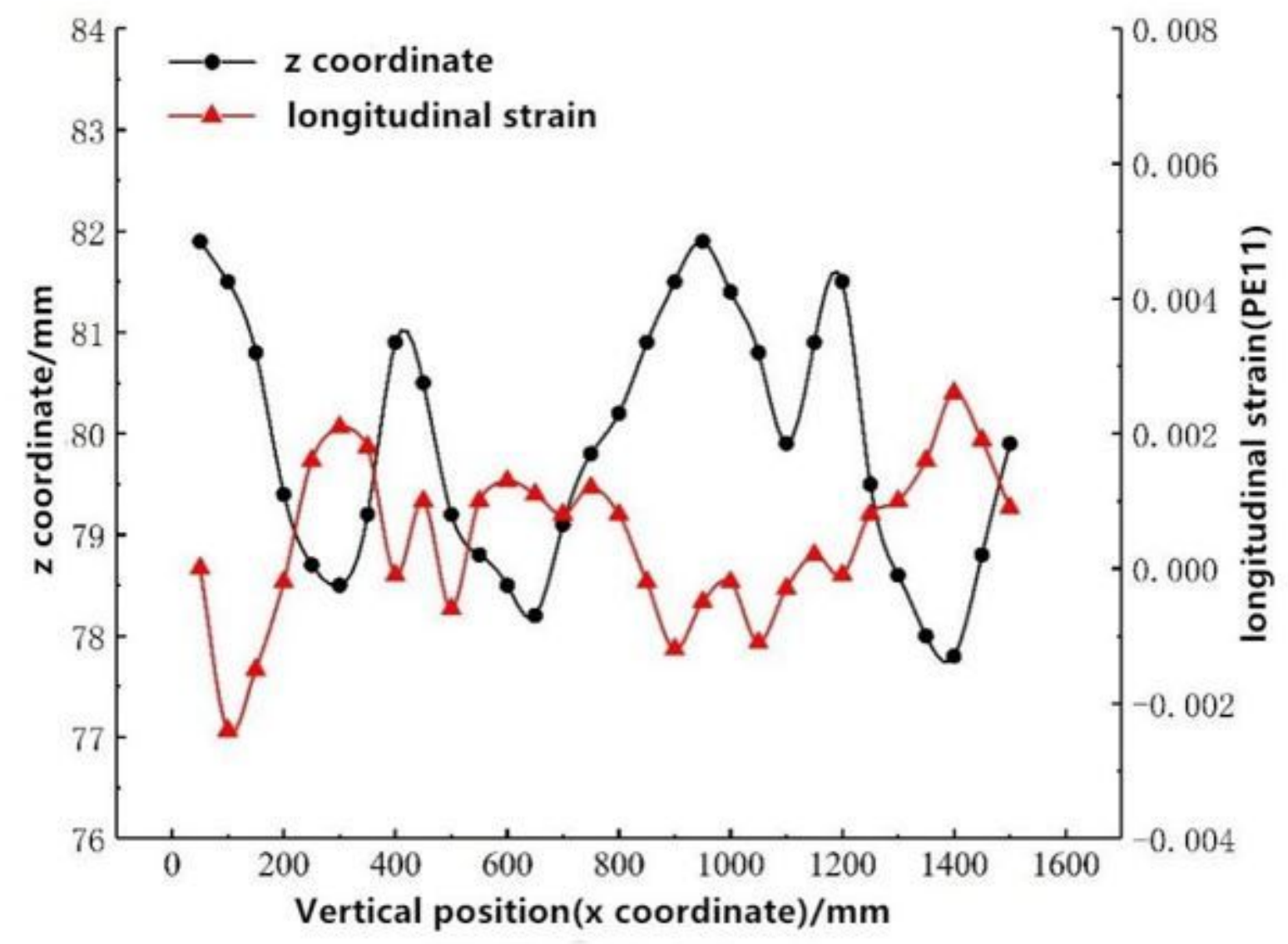

Figure 16

Curve of longitudinal strain, PE11, at flange edge as compared to the flange profile curve 


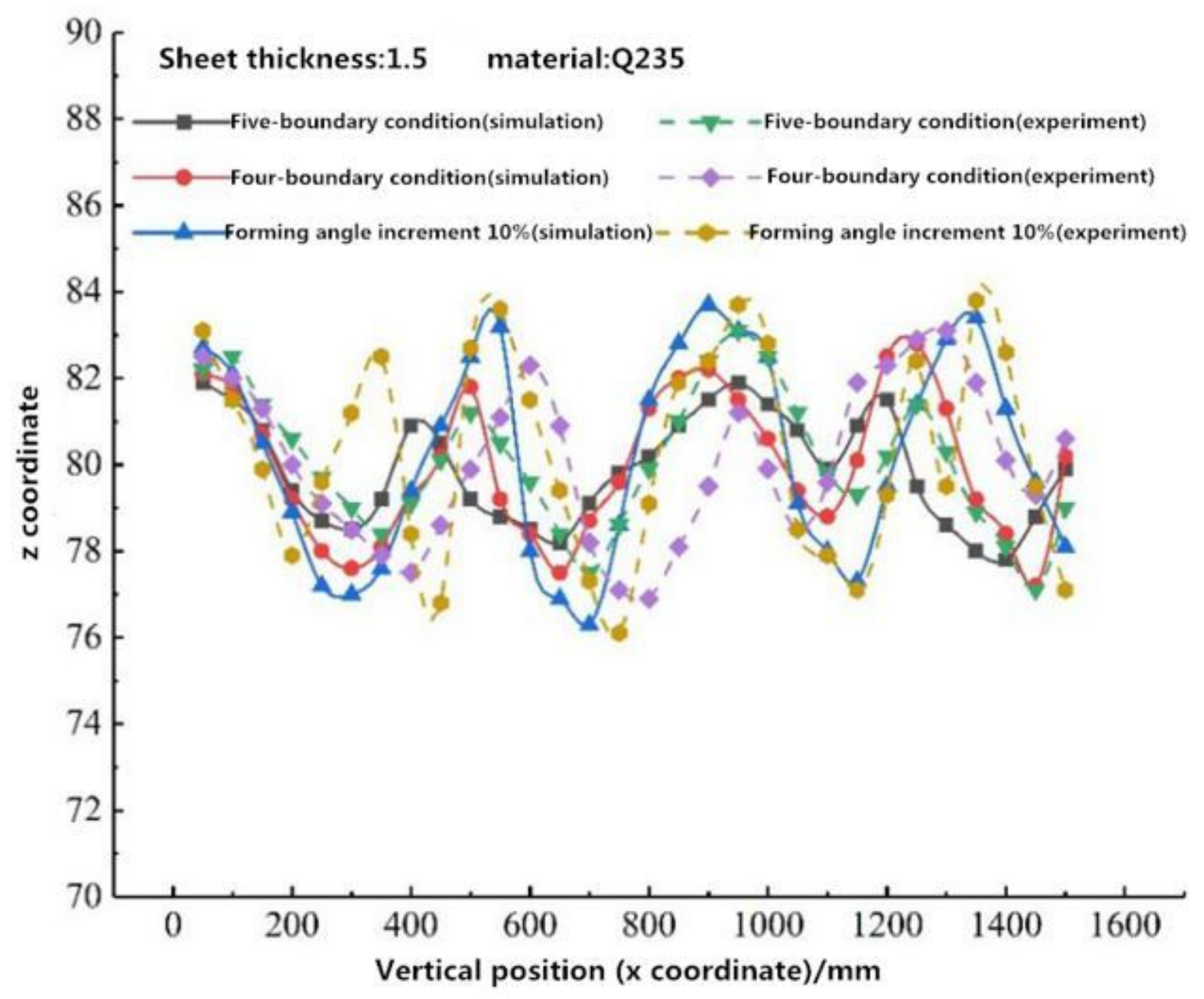

Figure 18

Comparison of experimental and simulated profiles of the flange edge under different forming angle distribution methods 


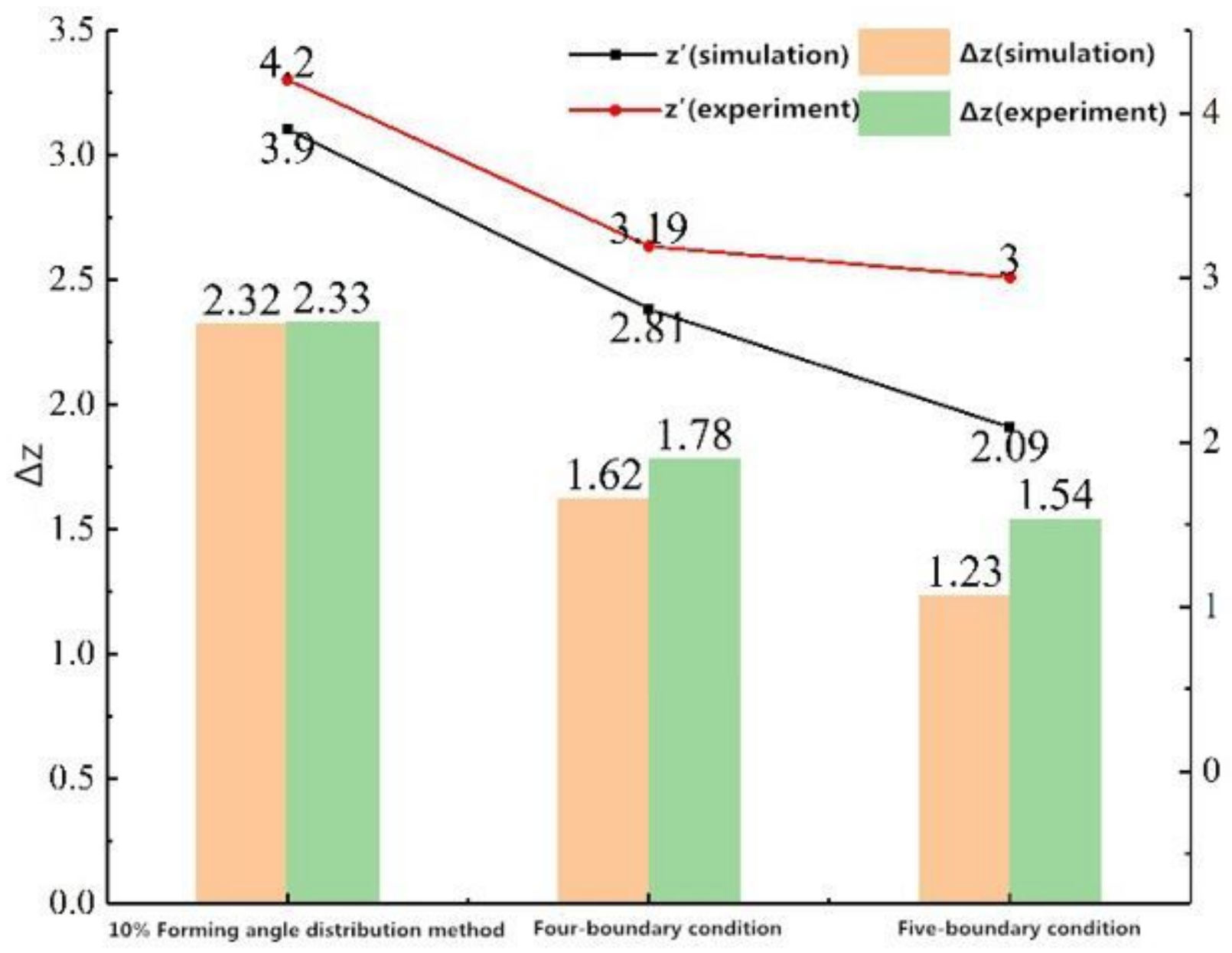

Forming angle distribution method

Figure 19

Comparison of experimental and simulated results of fluctuation of edge wave, $\Delta z$, and maximum deviation, $z$ ', of the edge under different forming angle distribution methods 


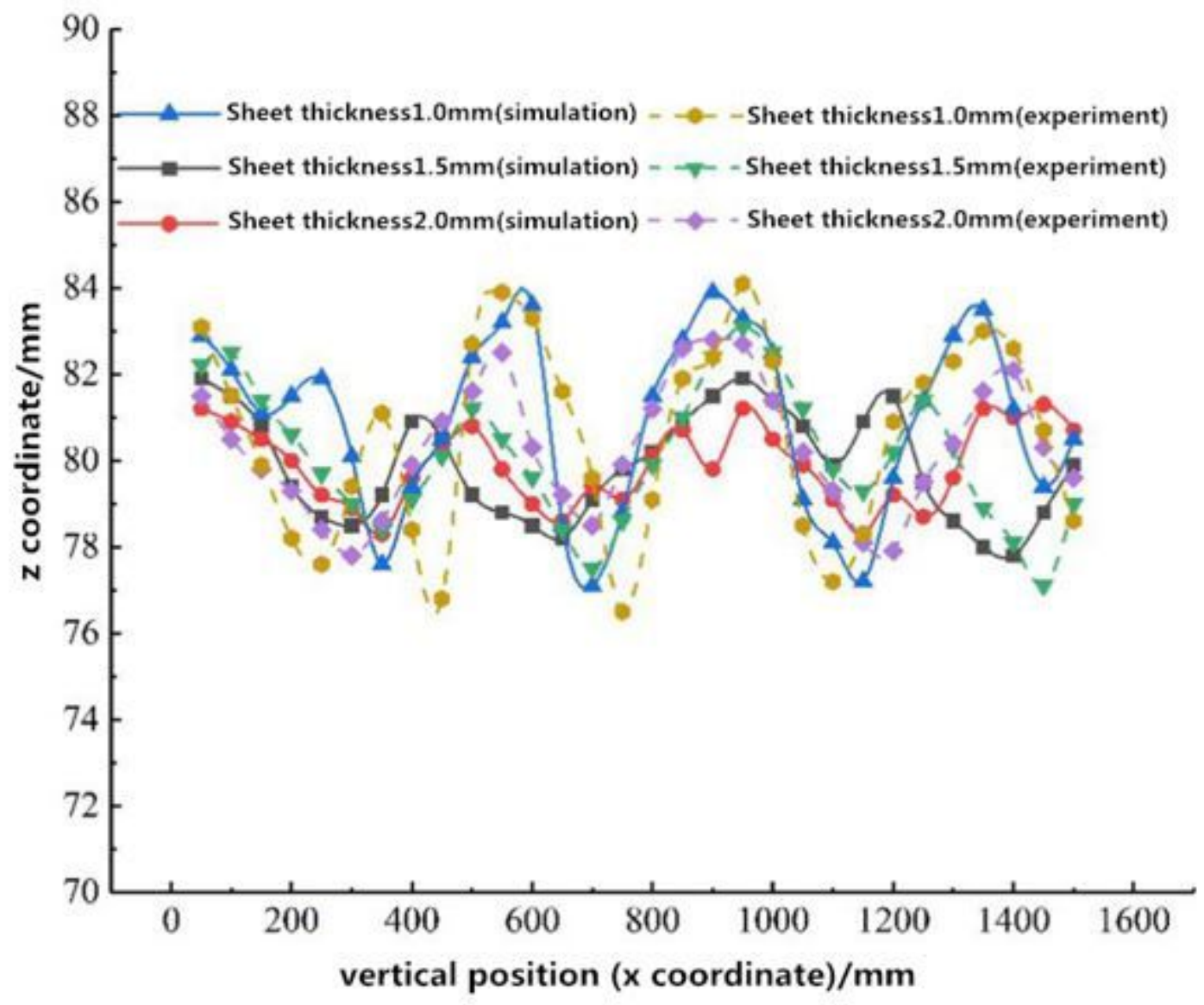

Figure 20

Comparison of resulting experimental and simulated profiles under different sheet thicknesses 


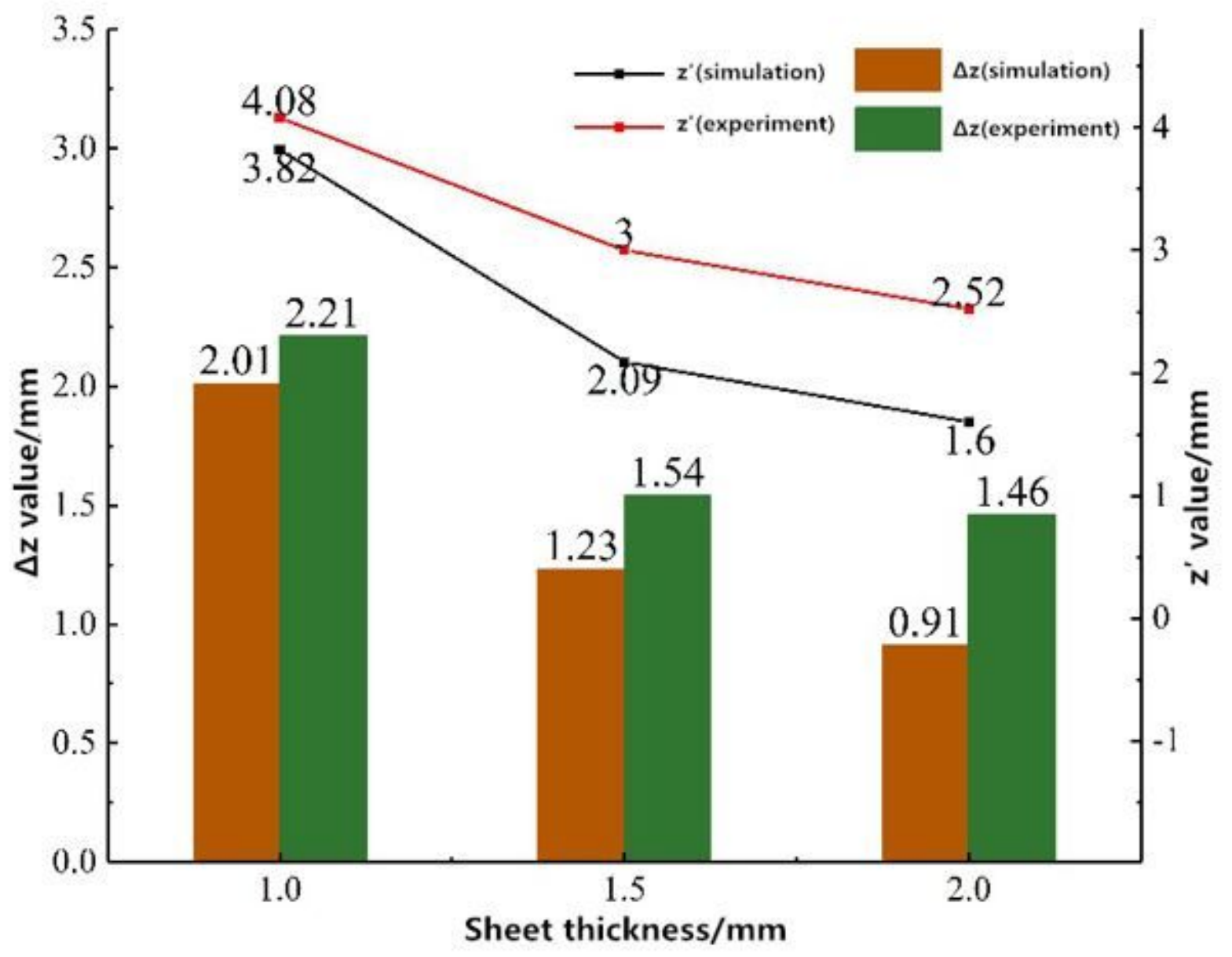

Figure 21

Comparison of experiment and simulation of fluctuation, $\Delta z$, and maximum deviation, $z^{\prime}$, of the flange edge under different sheet thicknesses 


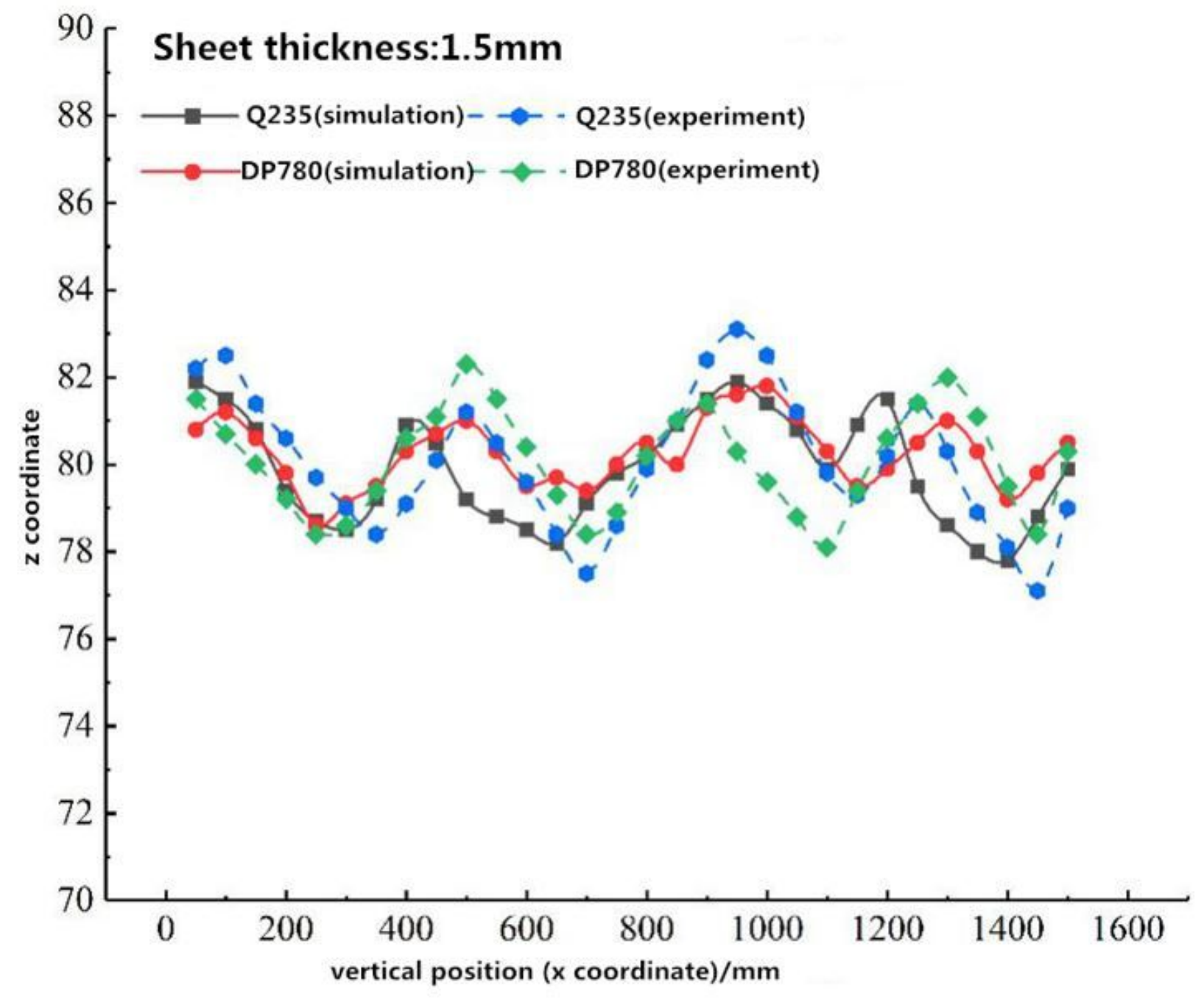

Figure 22

Comparison of curves From experimental and simulated profiles of the flange edge under different sheet yield strengths 


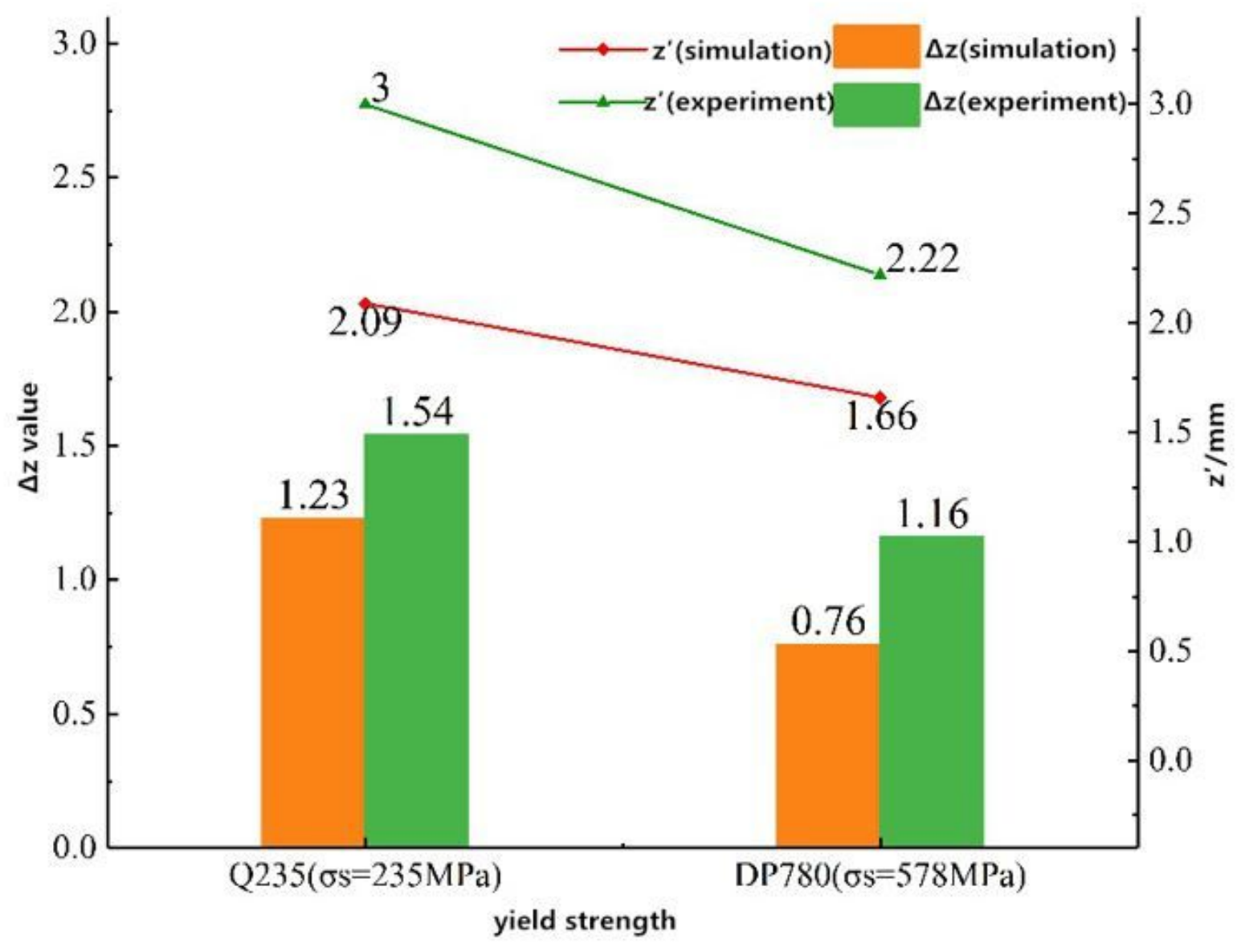

Figure 23

Comparison of experiment and simulation of fluctuation of edge wave, $\Delta z$, and maximum deviation, $z^{\prime}$, of the flange edge under different sheet yield strengths 